Published in final edited form as:

J Org Chem. 2019 May 17; 84(10): 6191-6198. doi:10.1021/acs.joc.9b00414.

\title{
Enantioselective Total Synthesis of (+)-Monocerin, a Dihydroisocoumarin Derivative with Potent Antimalarial Properties
}

\author{
Arun K. Ghosh ${ }^{\star}$ and Daniel S. Lee \\ Department of Chemistry and Department of Medicinal Chemistry, Purdue University, 560 Oval \\ Drive, West Lafayette, Indiana 47907
}

\begin{abstract}
We describe here the enantioselective synthesis of (+)-monocerin and its acetate derivative. The present synthesis features an efficient optically active synthesis of the $\beta$-hydroxy- $\gamma$-lactone derivative with high enantiomeric purity using a Sharpless dihydroxylation as the key step. The synthesis also highlights a tandem Lewis acid-catalyzed, oxocarbenium ion-mediated diastereoselective syn-allylation reaction and a methoxymethyl group promoted methylenation reaction. A selective $\mathrm{CrO}_{3}$-mediated oxidation of isochroman provided the corresponding lactone derivative. We investigated this reaction with a variety of Lewis acids. The synthesis is quite efficient and may be useful for the preparation of derivatives.
\end{abstract}

\section{Graphical Abstract}

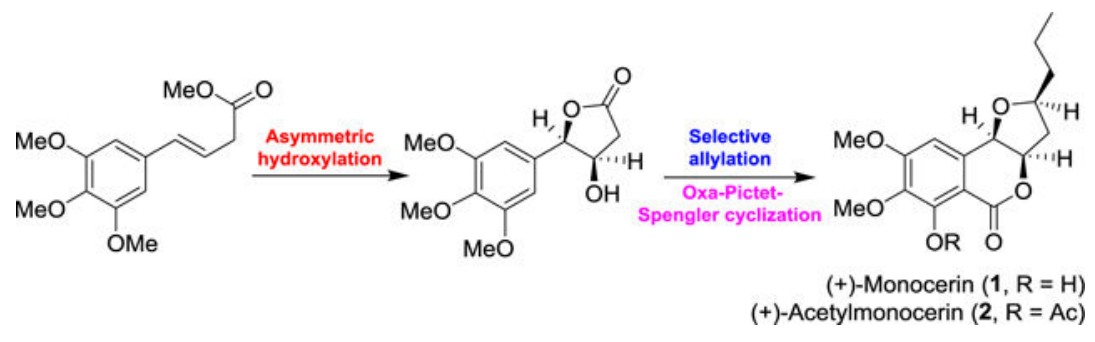

\section{Introduction}

Dihydrocoumarin and dihydroisocoumarin derivatives frequently occur in nature. ${ }^{1,2}$ These natural products exhibit a wide range of biological properties including antifungal, insecticidal, antiparasitic, and plant pathogenic activity. $3,4,5$ Monocerin (1, Figure 1), which was first isolated by Aldridge and Turner from Exserohilum monoceras (Drechsler), is a

\footnotetext{
*Corresponding Author akghosh@purdue.edu. ASSOCIATED CONTENT

Supporting Information

The supporting Information is available free of charge on the ACS Publications website http://pubs.acs.org.

${ }^{1} \mathrm{H}$ - and ${ }^{13} \mathrm{C}$-NMR spectra for all new compounds (PDF).

Conflicts of interest

There are no conflicts to declare.
} 
dihydroisocoumarin natural product which protects wheat against the powdery mildew, Erysiphe graminis. ${ }^{6}$ Aldridge and Turner also demonstrated monocerin's antifungal properties. Subsequently, Grove and Pople identified monocerin as a constituent of the entomogenous fungus, Fusarium larvarum Fuckel and showed its insecticidal properties. ${ }^{7}$ The assignment of absolute configuration of monocerin was addressed by Grove and pople as well as by Scott co-workers. ${ }^{7,8}$ Subsequently, synthesis and biosynthesis of the first PKSfree intermediate in monocerin was reported by Axford and co-workers. ${ }^{9}$ Since then, there have been many reports concerning the phytotoxic properties of monocerin and its derivatives 2-4. ${ }^{8,10-13}$ In 2008, Sappapan and co-workers reported potent antimalarial properties of monocerin against the multidrug-resistant strain of Plasmodium falcifarum with an $\mathrm{IC}_{50}$ value of $680 \mathrm{nM} .{ }^{14}$ Its acetate derivative 2 , also showed potent antimalarial activity $\left(\mathrm{IC}_{50}=820 \mathrm{nM}\right) .{ }^{14}$ Lasionectrin $(\mathbf{5})$, a dihydronaphthopyrone natural product was isolated from fermentations of the fungus Lasionectria (F-176,994). ${ }^{15}$ Like monocerin, Lasionectrin also exhibited antimalarial activity, however, subsequently weaker than that of monocerin. ${ }^{15}$ Monocerin features a 4-oxyisochroman-1-one structural unit and 2,3,5trisubstituted tetrahydrofuran ring containing all-cis stereochemistry.

Structural features as well as broad-spectrum biological properties of monocerin led to considerable synthetic attention over the years. Mori and co-workers reported the first synthesis of monocerin in racemic form. ${ }^{16}$ Simpson and co-workers then reported a biomimetic synthesis of monocerin. ${ }^{17}$ Since then, monocerin and its derivatives have attracted much synthetic interest due to their broad medicinal potentials. A number of total syntheses of monocerin has been reported. ${ }^{18-23}$ Recently, an enantioselective total synthesis of lasionectrin has also been reported. ${ }^{24}$ Herein, we report a short and practical synthesis of monocerin and its acetate derivative in optically active form. The synthesis features a Sharpless asymmetric dihydroxylation, a Lewis-acid catalyzed oxocarbenium ion-mediated stereoselective allylation, a formulation reaction, and $\mathrm{CrO}_{3}$ mediated oxidation of the benzopyran to a dihydroisocoumarin derivative. The synthesis is potentially amenable towards the synthesis of structural variants of monocerin for biological studies.

\section{Results and discussion}

Our synthetic strategy for (+)-monocerin is shown in Scheme 1. We planned an oxocarbenium ion-mediated allylation of acetate derivative 6 for stereoselective installation of the propyl chain of monocerin. We also envisioned an intramolecular oxocarbenium ionmediated formulation of the aromatic ring through the MOM-group to install the isochroman structure. We planned to investigate these transformations in a "one-pot" operation. A related C3-benzyloxy substituted five-membered ring oxocarbenium ion has been shown to promote allylation selectivity to provide the 1,3-cis product. ${ }^{25,26}$ The stereochemical outcome and selectivity with a methoxymethyl group is expected to be similar. The key allylation substrate can be synthesized from 3-hydroxy- $\gamma$-lactone 7 , which can be synthesized in an optically active manner by asymmetric dihydroxylation of $E$-olefin $\mathbf{8} .^{27,28}$ The requisite $E$-olefin can be synthesized by a stereoselective Wittig olefination using a $\gamma$ oxido-ylid and commercially available 3,4,5-trimethoxy benzaldehyde. ${ }^{29,30}$ 
Our synthesis of multigram quantities of $\boldsymbol{\gamma}$-lactone $\mathbf{7}$ and its derivatives is shown in Scheme 2, Wittig olefination of 3,4,5-trimethoxybenzaldehyde 9 was carried out with ylid generated from (2-carboxyethyl)triphenyl phosphonium bromide and potassium $t$-butoxide in THF at $-78{ }^{\circ} \mathrm{C}$ to $23{ }^{\circ} \mathrm{C}$ for $18 \mathrm{~h} .{ }^{29,31}$ The resulting $\beta, \gamma$-unsaturated acid was esterified by exposure to TMSCl in $\mathrm{MeOH}$ at $0{ }^{\circ} \mathrm{C}$ to $23{ }^{\circ} \mathrm{C}$ for $12 \mathrm{~h}$ to provide methyl ester 8 in $49 \%$ yield over 2steps. Asymmetric dihydroxylation of methyl ester 8 with AD-mix- $\beta$ in the presence of methanesulfonamide and sodium bicarbonate in aqueous $t$ - $\mathrm{BuOH}$ at $0{ }^{\circ} \mathrm{C}$ for $22 \mathrm{~h}$ afforded $\beta$-hydroxy- $\gamma$-lactone 7 in $90 \%$ yield. ${ }^{27}$ Optical purity of lactone 7 was over $95 \%$ ee as determined by chiral HPLC analysis using chiralpak IC column. Protection of the hydroxyl group as a MOM ether was achieved by reaction of lactone 7 with $\mathrm{MOMCl}$ in THF in the presence of diisopropylethylamine (DIPEA) and a catalytic amount of tetrabutylammonium iodide (TBAI) at $50{ }^{\circ} \mathrm{C}$ for $24 \mathrm{~h}$ to provide MOM-ether 10 in $95 \%$ yield. It was then converted to acetate derivative $\mathbf{6}$ in a two-step sequence, first, by DIBAL-H reduction in $\mathrm{CH}_{2} \mathrm{Cl}_{2}$ at $-78^{\circ} \mathrm{C}$ for $2 \mathrm{~h}$, followed by acetylation of the resulting crude lactol with acetic anhydride and triethylamine in the presence of a catalytic amount of DMAP in $\mathrm{CH}_{2} \mathrm{Cl}_{2}$ at $0{ }^{\circ} \mathrm{C}$ for $2 \mathrm{~h}$ to provide $\mathbf{6}$ as the major anomer along with a small amount of another anomer (ratio 14:1) in $88 \%$ combined yield over two-steps. Lactone 7 was converted to TBSprotected acetate derivative $\mathbf{1 1}$ by protection of the hydroxyl group as TBS ether followed by DIBAL-H reduction and acetylation as described above. Similarly, lactone 7 was also converted to MEM-derivative $\mathbf{1 2}$ as described above. The stereochemistry of the chiral center bearing acetate group for $\mathbf{6}, \mathbf{1 1}$ and $\mathbf{1 2}$ was assigned by using ${ }^{1} \mathrm{H}-\mathrm{NMR}$ NOESY experiments (Please see the Supporting Information).

With the synthesis of these acetates, we have investigated allylation promoted by various Lewis acids. We first carried out allylation of 6 with 1.1 equivalent of $\mathrm{SnBr}_{4}$ and 4 equivalent of allyltrimethylsilane in $\mathrm{CH}_{2} \mathrm{Cl}_{2}$ at $-78{ }^{\circ} \mathrm{C}$ for $3 \mathrm{~h}$. This resulted in a mixture (62: 38) of allyl derivatives 13a and 14a in $84 \%$ combined yield. The ratio was determined by ${ }^{1} \mathrm{H}$-NMR-analysis of diastereomeric protons. We then investigated other Lewis acids under various conditions and the results are shown in Table 1. The use of 2 equivalents of $\mathrm{SnBr}_{4}$ did not improve diastereoselectivity and the reaction yield was reduced (entry 2 ). The use of 1.1 equivalent of $\mathrm{TiCl}_{4}$ resulted in $69 \%$ yield of a mixture of diastereomers $(60: 40)$ similar to $\mathrm{SnBr}_{4}$ reaction (entry 3). An increase of Lewis acid to 2 equivalents, resulted in a decrease of reaction yields as well as a slight reduction of diastereomeric ratio (entry 4$)$. We also investigated the allylation reaction with $\mathrm{BF}_{3} \cdot \mathrm{OEt}_{2}$ and TMSOTf as the Lewis acids. In both cases, the reaction provided similar diastereomeric ratios and the reaction yields were $52 \%$ and $57 \%$, respectively (entries 5 and 6). We then examined allylation of TBS-protected acetate derivative 11 with 1.1 equivalent of $\mathrm{SnBr}_{4}$ and 4 equivalent of allyltrimethylsilane in $\mathrm{CH}_{2} \mathrm{Cl}_{2}$ at $-78{ }^{\circ} \mathrm{C}$ for $3 \mathrm{~h}$. This has resulted in a slight improvement in diastereomeric ratio ( $70: 30$ ) of allyl derivatives $\mathbf{1 3 b}$ and $\mathbf{1 4 b}$ in $81 \%$ yield (entry 7). The use of 1.1 equivalent of $\mathrm{TiCl}_{4}$ also provided comparable diastereoselectivity and yield (entry 8). Interestingly, allylation of MEM-protected acetate derivative 12 with 1.1 equivalent of $\mathrm{SnBr}_{4}$ and 4 equivalent of allyltrimethylsilane in $\mathrm{CH}_{2} \mathrm{Cl}_{2}$ at $-78{ }^{\circ} \mathrm{C}$ for $3 \mathrm{~h}$ resulted in allyl derivatives $13 \mathbf{c}$ and $14 \mathbf{c}$ in a 1:1 ratio and 33\% yield (entry 9). The reaction of $\mathbf{1 2}$ with 1.1 equivalent of $\mathrm{BF}_{3} \cdot \mathrm{OEt}_{2}$ and 1.5 equivalent of allyltrimethylsilane provided allyl derivatives $13 \mathbf{c}$ and $\mathbf{1 4 c}$ in a $63: 37$ ratio and the reaction yield was $36 \%$ (entry 10 ). While the observed 
diastereoselectivity of allylation was moderate, we assigned stereochemical identity of diastereomers $\mathbf{1 3 b}$ and $\mathbf{1 4 b}$ by using ${ }^{1} \mathrm{H}-\mathrm{NMR}$ NOESY experiments (Please see the Supporting Information for details).

The observed cis-diastereoselectivity is consistent with C3-alkoxy substituted tetrahydrofuranyl substrates examined by Worerpel and co-workers. ${ }^{25,26}$ However, the extent of diastereoselectivity of allylation is significantly lower presumably due to competing stereoelectronic effects. As shown in Figure 2, allylation of acetates can proceed through oxocarbenium ion intermediates $\mathbf{1 5}$ and 16. Intermediate $\mathbf{1 5}$ is preferred over $\mathbf{1 6}$ due to the pseudoequatorial orientation of the bulky trimethoxyphenyl group at the C4-position. ${ }^{26,32,33}$ The axial alkoxy group forms a gauche interaction with the aromatic group. Inside attack on the oxocarbenium ion intermediate $\mathbf{1 5}$ leads to major diastereomer 13. Intermediate 16 also shows a gauche interaction as well as 1,3-interactions between the bulky aromatic group and the $\mathrm{C} 2$-axial hydrogen. Changing of Lewis acids did not make much difference in selectivity. The size and nature of protecting groups also did not show much influence in diastereoselectivity. Moreover, the protecting groups remained unaffected under the reaction conditions.

We then investigated a Lewis acid-catalyzed allylation reaction at $-78{ }^{\circ} \mathrm{C}$ to $23^{\circ} \mathrm{C}$ in an effort to promote selective allylation, as well as to carry out Friedel-Crafts alkylation through the MOM or MEM groups on to the aromatic ring in a one-pot operation..$^{34,35}$ The results of these tandem allylation and Oxa-Pictet-Spengler cyclization are shown in Table 2. Initial reaction of MOM derivative 6 with 1.6 equivalent of $\mathrm{SnBr}_{4}$ in the presence of allyltrimethylsilane at $-78{ }^{\circ} \mathrm{C}$ to $23{ }^{\circ} \mathrm{C}$ for $3 \mathrm{~h}$ provided diastereomic mixture of allylated products 13a and 14a in 54\% yield as well as isochroman derivatives $\mathbf{1 7}$ and $\mathbf{1 8}$ in $22 \%$ yield (entry 1, Table 2). To promote complete formation of isochroman derivatives, we examined the reaction with additional equivalents of Lewis acid and the reaction was carried out for a longer period of time. Thus, reaction of MOM derivative $\mathbf{6}$ was carried out with 1.6 equivalent of $\mathrm{SnBr}_{4}$ in the presence of allyltrimethylsilane at $-78{ }^{\circ} \mathrm{C}$ to $23{ }^{\circ} \mathrm{C}$ for $4 \mathrm{~h}$. Reaction was monitored by TLC and showed complete consumption of the starting acetate derivatives. After this period, the reaction was cooled to $0{ }^{\circ} \mathrm{C}$ and 2 equivalents of $\mathrm{SnBr}_{4}$ was added, and the resulting mixture was warmed to $23{ }^{\circ} \mathrm{C}$ for $12 \mathrm{~h}$. This resulted in the formation of isochroman derivatives $\mathbf{1 7}$ and $\mathbf{1 8}$ in $52 \%$ yield. The diastereomeric ratio was determined to be $4: 1$ by ${ }^{1} \mathrm{H}$ NMR analysis (entry 2). Addition of additional equivalent of Lewis acid was then investigated at lower temperature. Acetate $\mathbf{6}$ was treated with 1.1 equivalent of $\mathrm{SnBr}_{4}$ at $-78{ }^{\circ} \mathrm{C}$ to $23{ }^{\circ} \mathrm{C}$ for $3 \mathrm{~h}$ and 2 equivalents of $\mathrm{SnBr}_{4}$ was added at $-78{ }^{\circ} \mathrm{C}$. The resulting mixture was allowed to warm from $-78{ }^{\circ} \mathrm{C}$ to $23^{\circ} \mathrm{C}$ for $12 \mathrm{~h}$. This reaction protocol furnished products $\mathbf{1 7}$ and $\mathbf{1 8}$ as a mixture (3.2:1) of diastereomers in $35 \%$ yield (entry 3 ). In a further optimization effort, acetate $\mathbf{6}$ was treated with 1.1 equivalent of $\mathrm{SnBr}_{4}$ at $-78{ }^{\circ} \mathrm{C}$ to for $3 \mathrm{~h}$ and then 2 equivalents of $\mathrm{SnBr}_{4}$ was added at $-78{ }^{\circ} \mathrm{C}$ and the reaction was warmed to $23^{\circ} \mathrm{C}$ for $12 \mathrm{~h}$. This has resulted products 17 and 18 as 3:1 mixture in $42 \%$ combined yield (entry 4). Oxa-Pictet-Spengler cyclization of acetate derivative 6 with $\mathrm{TiCl}_{4}$ in place of $\mathrm{SnBr}_{4}$ under similar reaction conditions, however, provided only trace amount of the products (entry 5). Interestingly, the reaction of MEM derivative 12 with $\mathrm{SnBr}_{4}$ and allyltrimethylsilane using conditions described in entry 2 
provided a mixture (1.7:1) of isochroman derivatives $\mathbf{1 7}$ and $\mathbf{1 8}$ in $21 \%$ combined yield (entry 6).

The synthesis of (+)-monocerin is shown in Scheme 3. MOM ether 6 was converted to allylated oxa Pictet-Spengler products $\mathbf{1 7}$ and $\mathbf{1 8}$. The diastereomers were separated by silica-gel chromatography and the major isomer $\mathbf{1 7}$ was hydrogenated under a hydrogenfilled balloon in the presence of a catalytic amount of $10 \%$ Pd-C in ethyl acetate at $23{ }^{\circ} \mathrm{C}$ for $12 \mathrm{~h}$ to provide saturated bicyclic ether 19 in $88 \%$ yield. The ${ }^{1} \mathrm{H}$-NMR and ${ }^{13} \mathrm{C}-\mathrm{NMR}$ spectra of our synthetic bicyclic ether 19 showed excellent agreement with reported derivative. ${ }^{18}$ Furthermore, NOESY correlation of cis-hydrogens in compound $\mathbf{1 7}$ supported stereochemistry of $\mathbf{1 7}$ and $\mathbf{1 9}$ (Please see the Supporting Information for details). Oxidation of the isochroman ring was carried out with $\mathrm{CrO}_{3}$ and pyridine in $\mathrm{CH}_{2} \mathrm{Cl}_{2}$ at $0{ }^{\circ} \mathrm{C}$ to $23{ }^{\circ} \mathrm{C}$ for $36 \mathrm{~h}$ to provide lactone derivative $\mathbf{2 0}$ in $40 \%$ yield (60\% brsm). To complete the synthesis of monocerin, selective deprotection of methyl ether was carried out by exposure to $\mathrm{BCl}_{3}$ in $\mathrm{CH}_{2} \mathrm{Cl}_{2}$ at $-10{ }^{\circ} \mathrm{C}$ for $2 \mathrm{~h}$ to provide synthetic (+)-monocerin, 1 in $41 \%$ yield (88\% brsm). The ${ }^{1} \mathrm{H}-$ and ${ }^{13} \mathrm{C}-\mathrm{NMR}$ spectra of synthetic (+)-monocerin $\left\{[\mathrm{a}]_{\mathrm{D}}{ }^{23}+57.8(c\right.$, $\left.\left.0.29, \mathrm{CHCl}_{3}\right)\right\}$ are in complete agreement with spectra reported for the natural (+)monocerin $\left\{[a]_{\mathrm{D}}{ }^{24}+53\left(c, 0.85, \mathrm{CHCl}_{3}\right)\right\} .{ }^{6} \mathrm{We}$ have also synthesized (+)-acetoxymonocerin 2 by treatment of 1 with acetic anhydride and pyridine in the presence of a catalytic amount of DMAP at $0{ }^{\circ} \mathrm{C}$ to $23{ }^{\circ} \mathrm{C}$ for $5 \mathrm{~h}$ to furnish acetate derivative $\mathbf{2}$ in $96 \%$ yield. $\left\{[\mathrm{a}]_{\mathrm{D}}{ }^{23}+3.6\right.$ $\left.\left(c, 0.29, \mathrm{CHCl}_{3}\right)\right\}$. Thus, (+)-monocerin 1 was synthesized in 10 steps in an overall $9 \%$ yield. The acetate derivative 2 was obtained in 11 steps in an overall $8.6 \%$ yield.

\section{Conclusions}

In summary, we have accomplished an enantioselective total synthesis of (+)-monocerin and its acetate derivative. The synthesis highlights a Lewis acid catalyzed tandem oxocarbenium ion-mediated stereoselective allylation and Friedel-Crafts type alkylation to provide the isochroman frame work in a one-pot operation. The allylation reaction was investigated with various Lewis acids and by varying protecting groups at the $\mathrm{C} 3$ position. While the stereochemical outcome for the major product has all cis-stereochemistry, the extent of diastereoselectivity was moderate due to competing stereoelectronic effects. The allylation substrate $\beta$-hydroxy- $\gamma$-lactone was synthesized conveniently in optically active form using Sharpless asymmetric dihydroxylation as the key step. The corresponding $E$-olefin was prepared selectivity by olefination with $\gamma$-oxido-ylid. Since monocerin and derivatives show a broad-range of biological activity, the current synthesis will provide access to structural variants in optically active form.

\section{Experimental Section}

All chemical and reagents were purchased from commercial suppliers and used without further purification unless otherwise noted. The following reaction solvents were distilled prior to use: $\mathrm{CH}_{2} \mathrm{Cl}_{2}$ from calcium hydride, diethyl ether and tetrahydrofuran from $\mathrm{Na} /$ benzophenone, methanol from activated magnesium under argon. All reactions were carried out under an argon atmosphere in either flame or oven-dried $\left(120^{\circ} \mathrm{C}\right)$ glassware. TLC analysis was conducted using glass-backed Thin-Layer Silica Gel Chromatography Plates 
(60 ̊, $250 \mu \mathrm{m}$ thickness, F-254 indicator). Column chromatography was performed using 230-400 mesh, $60 \AA$ pore diameter silica gel. ${ }^{1} \mathrm{H}-\mathrm{NMR}$ spectra were recorded at room temperature on a $400 \mathrm{MHz}$ spectrometer. ${ }^{13} \mathrm{C}$-NMR spectra were recorded on 100 and 200 $\mathrm{MHz}$ spectrometers. Chemical shifts ( $\delta$ values) are reported in parts per million and are referenced to the deuterated residual solvent peak. NMR data is reported as: $\delta$ value (chemical shift, $J$-value $(\mathrm{Hz})$, integration, where $\mathrm{s}=$ singlet, $\mathrm{d}=$ doublet, $\mathrm{t}=$ triplet, $\mathrm{q}=$ quartet, quint $=$ quintet, sep $=$ septet, $\mathrm{m}=$ multiplet, $\mathrm{dd}=$ doublet of doublets, $\mathrm{ddd}=$ doublet of doublet of doublets, $\mathrm{td}=$ triplet of doublets, $\mathrm{dq}=$ doublet of quartets, brs = broad singlet, app $=$ apparent). Optical rotations were recorded on a digital polarimeter. LRMS and HRMS spectra were recorded at the Purdue University Department of Chemistry Mass Spectrometry Center. These experiments were performed under ESI+ and positive atmosphere pressure chemical ionization (APCI+) conditions using an Orbitrap XL Instrument.

Methyl (E)-4-(3,4,5-Trimethoxyphenyl) But-3-enoate (8).-To a cooled suspension of (2-carboxyethyl) triphenylphosphonium bromide ( $2.99 \mathrm{~g}, 7.2 \mathrm{mmol}, 1.2$ equiv) and 3,4,5trimethoxybenzaldehyde $\left(1.18 \mathrm{~g}, 6 \mathrm{mmol}, 1.0\right.$ equiv) in THF $(45 \mathrm{~mL})$ at $-78^{\circ} \mathrm{C}$ was slowly added a solution of $t$-BuOK $(15 \mathrm{~mL}, 15 \mathrm{mmol}, 1.0 \mathrm{THF}$ solution, 2.5 equiv). After addition, the mixture was stirred at $-78{ }^{\circ} \mathrm{C}$ for $1 \mathrm{~h}$ and was then warmed to $23^{\circ} \mathrm{C}$ overnight. The resulting suspension was concentrated under reduced pressure, $\mathrm{H}_{2} \mathrm{O}(100 \mathrm{~mL})$ was added, and the mixture was washed with $\mathrm{CH}_{2} \mathrm{Cl}_{2}$. The aqueous phase was acidified to $\mathrm{pH}=1$ with a $1 \mathrm{M}$ solution of $\mathrm{HCl}$ and extracted with $\mathrm{Et}_{2} \mathrm{O}(3 \mathrm{x})$. The combined organic extracts were dried over $\mathrm{MgSO}_{4}$, filtered and concentrated under reduced pressure.

The crude $\beta, \gamma$-unsaturated carboxylic acid above was then dissolved in distilled $\mathrm{MeOH}$ (20 $\mathrm{mL})$ and $\mathrm{TMSCl}\left(0.97 \mathrm{ml}, 1.26\right.$ equiv) was dropwise added at $0{ }^{\circ} \mathrm{C}$ under argon atmosphere. The mixture was stirred at $23{ }^{\circ} \mathrm{C}$ overnight then concentrated under reduced pressure. The crude product was purified by silica gel column chromatography (10\% EtOAc in hexanes) to yield 8 (746 mg, $49 \%$ over two steps) as a yellow oil. ${ }^{1} \mathrm{H}$ NMR $\left(400 \mathrm{MHz}, \mathrm{CDCl}_{3}\right) \delta 6.58$ (s, 2H), $6.41(\mathrm{~d}, J=15.8,1 \mathrm{H}), 6.20(\mathrm{dt}, J=15.8,7.1 \mathrm{~Hz}, 1 \mathrm{H}), 3.86(\mathrm{~s}, 6 \mathrm{H}), 3.83(\mathrm{~s}, 3 \mathrm{H})$, $3.71(\mathrm{~s}, 3 \mathrm{H}), 3.24(\mathrm{dd}, J=7.1,1.5 \mathrm{~Hz}, 2 \mathrm{H}) ;{ }^{13} \mathrm{C}\left\{{ }^{1} \mathrm{H}\right\} \mathrm{NMR}\left(100 \mathrm{MHz}, \mathrm{CDCl}_{3}\right)$ 8: 171.9, 153.2, 137.7, 133.3, 132.5, 121.0, 103.3, 60.8, 56.0, 51.9, 38.0. LRMS (ESI) $267.0([\mathrm{M}+\mathrm{H}]$ ${ }^{+}$); HRMS (ESI-Orbitrap) m/z: [M+Na] ${ }^{+}$calcd for $\mathrm{C}_{14} \mathrm{H}_{18} \mathrm{O}_{5} \mathrm{Na}$; [M+Na] $]^{+}$: 289.1046, found 289.1049 .

(4R,5R)-4-Hydroxy-5-(3,4,5-Trimethoxyphenyl)Dihydrofuran-2(3H)-one (7)-AD-mix- $\beta$ (3.926 g), $\mathrm{NaHCO}_{3}$ (706 mg, $8.4 \mathrm{mmol}$ ), and methanesulfonamide (267 mg, 2.8 mmol) were dissolved in $t$ - $\mathrm{BuOH}(7 \mathrm{~mL})$ and water $(14 \mathrm{~mL})$. After the mixture was cooled to $0{ }^{\circ} \mathrm{C}$, methyl ester $8(746 \mathrm{mg}, 2.8 \mathrm{mmol})$ in $t$ - $\mathrm{BuOH}(7 \mathrm{~mL})$ was added. The mixture was stirred at $0{ }^{\circ} \mathrm{C}$ for $22 \mathrm{~h}$. After this period, sodium sulfite $(5.0 \mathrm{~g})$ was added. The mixture was stirred for $1 \mathrm{~h}$ at $23{ }^{\circ} \mathrm{C}$ and extracted with EtOAc (3x). The combined organic layer was dried with $\mathrm{Na}_{2} \mathrm{SO}_{4}$, filtered, and concentrated under reduced pressure. The crude product was then purified by column chromatography on silica gel (50-80\% EtOAc in hexanes) to afford lactone 7 (700 mg, $90 \%)$ as a white amorphous solid. [a $]_{\mathrm{D}}{ }^{20}-23.9\left(c 0.41, \mathrm{CHCl}_{3}\right)$; ${ }^{1} \mathrm{H}$ NMR $\left(400 \mathrm{MHz}, \mathrm{CDCl}_{3}\right)$ \&: $6.54(\mathrm{~s}, 2 \mathrm{H}), 5.38(\mathrm{~d}, J=3.3 \mathrm{~Hz}, 1 \mathrm{H}), 4.62-4.56(\mathrm{~m}, 1 \mathrm{H})$, 
$3.82(\mathrm{~s}, 6 \mathrm{H}), 3.78(\mathrm{~s}, 3 \mathrm{H}), 2.85$ (ddd, $J=17.5,5.0,1.5 \mathrm{~Hz}, 1 \mathrm{H}), 2.71(\mathrm{~d}, J=17.5 \mathrm{~Hz}, 1 \mathrm{H})$,

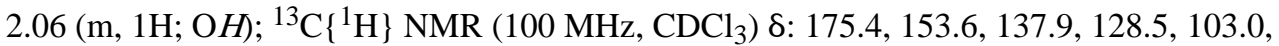
85.1, 70.1, 60.7, 56.1, 38.4.LRMS (ESI) $269.0[\mathrm{M}+\mathrm{H}]^{+}$; HRMS (ESI-Orbitrap) m/z: [M+H] + calcd for $\mathrm{C}_{13} \mathrm{H}_{16} \mathrm{O}_{6}+\mathrm{H} 269.1020$, found 269.1024.

(4R,5R)-4-(Methoxymethoxy)-5-(3,4,5-Trimethoxyphenyl)Dihydrofuran-2(3H)one (10a).-To a stirred solution of lactone 7 (272 mg, $1.02 \mathrm{mmol}$ ) in distilled THF (4 mL) at $0{ }^{\circ} \mathrm{C}$ under argon atmosphere were consecutively added DIPEA $(1.77 \mathrm{~mL}, 10.2 \mathrm{mmol})$, TBAI ( $75 \mathrm{mg}, 0.20 \mathrm{mmol})$, and MOM-Cl $(0.44 \mathrm{~mL}, 5.80 \mathrm{mmol})$. The reaction mixture was then stirred at $50{ }^{\circ} \mathrm{C}$ for $24 \mathrm{~h}$. After dilution with $\mathrm{CH}_{2} \mathrm{Cl}_{2}$, the mixture was washed with water, dried over $\mathrm{Na}_{2} \mathrm{SO}_{4}$, filtered, and concentrated under reduced pressure. The crude residue was purified by column chromatography on silica gel (60\% EtOAc in hexane) to give MOM protected lactone 10a (300 mg, 95\%) as a yellow amorphous solid. [a $]_{\mathrm{D}}{ }^{20}-46.1$ (c 0.79, $\left.\mathrm{CHCl}_{3}\right) ;{ }^{1} \mathrm{H} \mathrm{NMR}\left(400 \mathrm{MHz}, \mathrm{CDCl}_{3}\right) \delta: 6.58(\mathrm{~d}, J=0.6 \mathrm{~Hz}, 2 \mathrm{H}), 5.43(\mathrm{~d}, J=3.8$ $\mathrm{Hz}, 1 \mathrm{H}), 4.56$ (ddd, $J=5.1,3.8,0.9 \mathrm{~Hz}, 1 \mathrm{H}), 4.32(\mathrm{~d}, J=7.1 \mathrm{~Hz}, 1 \mathrm{H}), 4.14$ (d, $J=7.1 \mathrm{~Hz}$, $1 \mathrm{H}), 3.85(\mathrm{~s}, 6 \mathrm{H}), 3.83(\mathrm{~s}, 3 \mathrm{H}), 3.06(\mathrm{~s}, 3 \mathrm{H}), 2.88(\mathrm{dd}, J=17.5,5.3 \mathrm{~Hz}, 1 \mathrm{H}), 2.73(\mathrm{dd}, J=$ $17.5,0.9 \mathrm{~Hz}, 1 \mathrm{H}) ;{ }^{13} \mathrm{C}\left\{{ }^{1} \mathrm{H}\right\} \mathrm{NMR}\left(100 \mathrm{MHz}, \mathrm{CDCl}_{3}\right) \delta: 174.9,153.2,137.9,129.3,103.6$, 95.1, 84.6, 74.1, 60.8, 56.1, 55.4, 37.5. LRMS (ESI) 647.2 [2M+Na] ${ }^{+}$; HRMS (ESIOrbitrap) m/z: $[\mathrm{M}+\mathrm{Na}]^{+}$calcd for $\mathrm{C}_{15} \mathrm{H}_{20} \mathrm{O}_{7} \mathrm{Na} 335.1101$, found 335.1103 .

(4R,5R)-4-(Methoxymethoxy)-5-(3,4,5-Trimethoxyphenyl)Tetrahydrofuran-2-ol (6).-To a stirred solution of lactone $10 a(240 \mathrm{mg}, 0.77 \mathrm{mmol})$ in $\mathrm{CH}_{2} \mathrm{Cl}_{2}(6 \mathrm{~mL})$ at $-78{ }^{\circ} \mathrm{C}$ under argon atmosphere was added DIBAL-H $(0.92 \mathrm{~mL}, 0.92 \mathrm{mmol})$ and the resulting mixture was stirred at the same temperature for $2 \mathrm{~h}$. After this period, the reaction mixture was quenched by the addition of $\mathrm{MeOH}(3 \mathrm{~mL})$ and warmed to $23^{\circ} \mathrm{C}$. Then, saturated aqueous solution of sodium potassium tartarate was added and stirred vigorously at $23^{\circ} \mathrm{C}$ for $2 \mathrm{~h}$ until it forms white suspension. The suspension was filtered through a plug of Celite and solvents were removed under reduced pressure.

To a crude lactol, DMAP (17 mg, $0.14 \mathrm{mmol}), \mathrm{Et}_{3} \mathrm{~N}(0.50 \mathrm{~mL}, 3.59 \mathrm{mmol})$ and $\mathrm{Ac}_{2} \mathrm{O}(0.17$ $\mathrm{mL}, 1.80 \mathrm{mmol}$ ) were added at $0{ }^{\circ} \mathrm{C}$ under argon atmosphere and the resulting mixture was stirred for $2 \mathrm{~h}$. Upon completion, solvents were removed under reduced pressure and the crude product was purified by column chromatography over silica gel (50\% EtOAc in hexanes) to give acetate $\mathbf{6}(240 \mathrm{mg})$ as the major isomer and the corresponding minor anomer $(16 \mathrm{mg})$ as colorless oils in $88 \%$ over two steps. Acetate 6: ${ }^{1} \mathrm{H}$ NMR $(400 \mathrm{MHz}$, $\mathrm{CDCl}_{3}$ ) $\delta: 6.61(\mathrm{~s}, 1 \mathrm{H}), 6.59$ (dd, $\left.J=6.1,3.3 \mathrm{~Hz}, 1 \mathrm{H}\right), 5.09$ (d, $\left.J=3.8 \mathrm{~Hz}, 1 \mathrm{H}\right), 4.45$ (ddd, $J$ $=5.8,3.8,1.9 \mathrm{~Hz}, 1 \mathrm{H}), 4.30(\mathrm{~d}, J=7.0 \mathrm{~Hz}, 1 \mathrm{H}), 4.12(\mathrm{~d}, J=6.9 \mathrm{~Hz}, 1 \mathrm{H}), 3.84(\mathrm{~s}, 6 \mathrm{H}), 3.81$ (s, $3 \mathrm{H}), 3.06$ (s, 3H), 2.53 (ddd, $J=14.7,6.1,1.9 \mathrm{~Hz}, 1 \mathrm{H}), 2.37$ (ddd, $J=14.7,6.1,3.3 \mathrm{~Hz}$, 1H), 2.06 (s, 3H); ${ }^{13} \mathrm{C}\left\{{ }^{1} \mathrm{H}\right\}$ NMR (Major anomer, $101 \mathrm{MHz}, \mathrm{CDCl}_{3}$ ) $\delta: 170.2,152.9,137.5$, 131.1, 104.2, 97.4, 95.1, 83.7, 76.6, 60.8, 56.1, 55.2, 40.4, 21.2; LRMS (ESI) 735.2 ([2M $+\mathrm{Na}]^{+}$); HRMS (ESI-Orbitrap) m/z: [M+Na] ${ }^{+}$calcd for $\mathrm{C}_{17} \mathrm{H}_{24} \mathrm{O}_{8} \mathrm{Na} 379.1363$, found 379.1360; Minor Isomer: ${ }^{1} \mathrm{H}$ NMR (400 MHz, CDCl3) $\delta: 6.63(\mathrm{~s}, 2 \mathrm{H}), 6.42(\mathrm{~d}, \mathrm{~J}=5.8 \mathrm{~Hz}$, $1 \mathrm{H}), 5.08(\mathrm{~d}, \mathrm{~J}=4.5 \mathrm{~Hz}, 1 \mathrm{H}), 4.42-4.35(\mathrm{~m}, 2 \mathrm{H}), 4.23-4.17(\mathrm{~m}, 1 \mathrm{H}), 3.88-3.85(\mathrm{~s}, 6 \mathrm{H})$, $3.83(\mathrm{~s}, \mathrm{~J}=0.9 \mathrm{~Hz}, 3 \mathrm{H}), 3.11(\mathrm{~s}, 3 \mathrm{H}), 2.50-2.42(\mathrm{~m}, 1 \mathrm{H}), 2.40-2.34(\mathrm{~m}, 1 \mathrm{H}), 2.13(\mathrm{~s}, 3 \mathrm{H})$; 
13C $\left\{{ }^{1} \mathrm{H}\right\}$ NMR (101 MHz, CDCl3) $\delta: 170.4,152.8,132.14,129.8,104.4,98.0,95.2,86.4$, 75.4, 60.8, 56.0, 55.2, 39.3, 21.4; LRMS (ESI) $357.1\left([\mathrm{M}+\mathrm{H}]^{+}\right)$.

(4R,5R)-4-(Methoxymethoxy)-5-(3,4,5-Trimethoxyphenyl)Tetrahydrofuran-2-yl Acetate (11).-To a stirred solution of lactone $7(95 \mathrm{mg}, 0.354 \mathrm{mmol})$ in $\mathrm{CH}_{2} \mathrm{Cl}_{2}(3 \mathrm{~mL})$ at $0{ }^{\circ} \mathrm{C}$ under argon atmosphere were added 2,6-lutidine $(0.12 \mathrm{~mL}, 1.06 \mathrm{mmol})$ and TBSOTf $(0.12 \mathrm{~mL}, 0.53 \mathrm{mmol})$. The reaction mixture was warmed to $23^{\circ} \mathrm{C}$ and stirred for $3 \mathrm{~h}$. When the reaction was finished, the mixture was quenched by the addition of saturated aqueous $\mathrm{NaHCO}_{3}$ and extracted with dichloromethane. The extracts were dried over $\mathrm{Na}_{2} \mathrm{SO}_{4}$, filtered, and concentrated under reduced pressure. The crude product was purified by silica gel column chromatography (40\% EtOAc in hexanes) to give silyl ether derivative (69 $\mathrm{mg}$, $51 \%)$ as an orange amorphous solid. $[a]_{\mathrm{D}}{ }^{20}-34.7\left(c 0.29, \mathrm{CHCl}_{3}\right) ;{ }^{1} \mathrm{H} \mathrm{NMR}(400 \mathrm{MHz}$, $\left.\mathrm{CDCl}_{3}\right) \delta: 6.54(\mathrm{~s}, 2 \mathrm{H}), 5.37(\mathrm{~d}, \mathrm{~J}=3.5 \mathrm{~Hz}, 1 \mathrm{H}), 4.55(\mathrm{ddd}, \mathrm{J}=4.8,3.5,0.7 \mathrm{~Hz}, 1 \mathrm{H}), 3.85(\mathrm{~s}$, $6 \mathrm{H}), 3.82(\mathrm{~s}, 3 \mathrm{H}), 2.87(\mathrm{dd}, \mathrm{J}=17.1,4.8 \mathrm{~Hz}, 1 \mathrm{H}), 2.59(\mathrm{dd}, \mathrm{J}=17.1,0.7 \mathrm{~Hz}, 1 \mathrm{H}), 0.67(\mathrm{~s}$, $9 \mathrm{H}),-0.13(\mathrm{~s}, 3 \mathrm{H}),-0.34(\mathrm{~s}, 3 \mathrm{H}) ;{ }^{13} \mathrm{C}\left\{{ }^{1} \mathrm{H}\right\}$ NMR $\left(100 \mathrm{MHz}, \mathrm{CDCl}_{3}\right) \delta 175.5,153.1,138.0$, 129.9, 103.8, 86.0, 71.5, 60.8, 56.1, 40.2, 25.3, 17.7, -5.4, -5.6; LRMS (ESI) 405.2 [M+Na] + .

The title compound was prepared from above lactone $(55 \mathrm{mg})$ was reduced and protected as acetate derivatives following the procedure described for the preparation of acetate $\mathbf{6}$. The compound was purified by flash column chromatography over silica gel (20\% EtOAc in hexanes) to give acetate $\mathbf{1 1}$ (50 mg, 81\% over two steps) as a white amorphous solid. [a] $]_{\mathrm{D}}{ }^{20}$ - 13.6 ( c 0.5, $\left.\mathrm{CHCl}_{3}\right) ;{ }^{1} \mathrm{H} \mathrm{NMR}\left(400 \mathrm{MHz}, \mathrm{CDCl}_{3}\right) \delta$ : $6.60(\mathrm{dd}, \mathrm{J}=5.9,3.8 \mathrm{~Hz}, 1 \mathrm{H}), 6.56$ $(\mathrm{d}, \mathrm{J}=0.5 \mathrm{~Hz}, 2 \mathrm{H}), 5.03(\mathrm{~d}, \mathrm{~J}=3.6 \mathrm{~Hz}, 1 \mathrm{H}), 4.43(\mathrm{ddd}, \mathrm{J}=5.4,3.6,1.9 \mathrm{~Hz}, 1 \mathrm{H}), 3.83(\mathrm{~s}$, $6 \mathrm{H}), 3.78(\mathrm{~s}, 3 \mathrm{H}), 2.44-2.27(\mathrm{~m}, 2 \mathrm{H}), 2.05(\mathrm{~s}, 3 \mathrm{H}), 0.67$ (s, 9H), -0.17 (s, 3H), -0.35 (s, $3 \mathrm{H}) ;{ }^{13} \mathrm{C}\left\{{ }^{1} \mathrm{H}\right\}$ NMR $\left(100 \mathrm{MHz}, \mathrm{CDCl}_{3}\right) \delta: 170.3,152.7,137.5,131.7,104.6,97.9,85.2$, 73.7, 60.7, 56.0, 43.1, 25.4, 21.2, 17.8, -5.4, -5.5. LRMS (ESI) 449.2 [M+Na] ${ }^{+}$; HRMS (ESI-Orbitrap) m/z: [M+Na] ${ }^{+}$calcd for $\mathrm{C}_{21} \mathrm{H}_{34} \mathrm{O}_{7} \mathrm{SiNa} 449.1966$, found 449.1969 .

(4R,5R)-4-((2-Methoxyethoxy)Methoxy)-5-(3,4,5Trimethoxyphenyl)Tetrahydrofuran-2-yl Acetate (12).-To a stirred solution of lactone $7(125 \mathrm{mg}, 0.47 \mathrm{mmol})$ in distilled THF $(2 \mathrm{~mL})$ at $0{ }^{\circ} \mathrm{C}$ under argon atmosphere were consecutively added DIPEA $(0.81 \mathrm{~mL}, 4.7 \mathrm{mmol})$, TBAI $(43 \mathrm{mg}, 0.12 \mathrm{mmol})$ and MEM-Cl $(0.27 \mathrm{~mL}, 2.33 \mathrm{mmol})$. The resulting reaction mixture was stirred at $55{ }^{\circ} \mathrm{C}$ for 72 h. Upon completion, the mixture was diluted with EtOAc and washed with water. The organic phase was dried over $\mathrm{Na}_{2} \mathrm{SO}_{4}$, filtered, and concentrated under reduced pressure. The crude product was purified by column chromatography on silica gel $(80 \%$ EtOAc in hexanes) to give MEM protected lactone (131 $\mathrm{mg}, 79 \%)$ as a yellow amorphous solid. $[\mathrm{a}]_{\mathrm{D}}{ }^{20}-25.5\left(c 0.29, \mathrm{CHCl}_{3}\right) ;{ }^{1} \mathrm{H} \mathrm{NMR}\left(400 \mathrm{MHz}, \mathrm{CDCl}_{3}\right) \delta: 6.57$ (s, 2H), $5.43(\mathrm{~d}, \mathrm{~J}=3.8$ $\mathrm{Hz}, 1 \mathrm{H}), 4.62(\mathrm{ddd}, \mathrm{J}=5.0,3.8,1.0 \mathrm{~Hz}, 1 \mathrm{H}), 4.42(\mathrm{~d}, \mathrm{~J}=7.3 \mathrm{~Hz}, 1 \mathrm{H}), 4.23(\mathrm{~d}, \mathrm{~J}=7.3 \mathrm{~Hz}$, $1 \mathrm{H}), 3.85(\mathrm{~s}, 6 \mathrm{H}), 3.83(\mathrm{~s}, 3 \mathrm{H}), 3.45(\mathrm{ddd}, \mathrm{J}=10.6,5.6,3.3 \mathrm{~Hz}, 1 \mathrm{H}), 3.41-3.36(\mathrm{~m}, 2 \mathrm{H})$, $3.32(\mathrm{~s}, 3 \mathrm{H}), 3.22(\mathrm{ddd}, \mathrm{J}=10.6,5.3,3.4 \mathrm{~Hz}, 1 \mathrm{H}), 2.87(\mathrm{dd}, \mathrm{J}=17.6,5.2 \mathrm{~Hz}, 1 \mathrm{H}), 2.74(\mathrm{dd}$, $\mathrm{J}=17.5,1.0 \mathrm{~Hz}, 1 \mathrm{H}) ;{ }^{13} \mathrm{C}\left\{{ }^{1} \mathrm{H}\right\} \mathrm{NMR}\left(100 \mathrm{MHz}, \mathrm{CDCl}_{3}\right) \delta: 175.0,153.2,137.9,129.3$, 103.5, 93.9, 84.6, 74.0, 71.4, 67.0, 60.8, 58.9, 56.1, 37.4; LRMS (ESI) $379.4[\mathrm{M}+\mathrm{Na}]^{+}$. 
The title compound was prepared from above lactone $(111 \mathrm{mg})$ following the procedure described for the preparation of acetate $\mathbf{6}$. The compound was purified by flash column chromatography (20\% EtOAc in hexane) to give acetate $\mathbf{1 2}$ (50 mg, $72 \%$ over two steps) as a white amorphous solid. $[a]_{\mathrm{D}}{ }^{20}+4.1\left(c 0.69, \mathrm{CHCl}_{3}\right) ;{ }^{1} \mathrm{H} \mathrm{NMR}(400 \mathrm{MHz}, \mathrm{CDCl} 3) \delta$ : $6.58(\mathrm{~s}, 2 \mathrm{H}), 6.55(\mathrm{dd}, \mathrm{J}=6.1,3.3 \mathrm{~Hz}, 1 \mathrm{H}), 5.07(\mathrm{~d}, \mathrm{~J}=3.8 \mathrm{~Hz}, 1 \mathrm{H}), 4.48$ (ddd, J = 5.8, 3.8, $1.7 \mathrm{~Hz}, 1 \mathrm{H}), 4.37(\mathrm{~d}, \mathrm{~J}=7.2 \mathrm{~Hz}, 1 \mathrm{H}), 4.19(\mathrm{~d}, \mathrm{~J}=7.1 \mathrm{~Hz}, 1 \mathrm{H}), 3.81(\mathrm{~s}, 6 \mathrm{H}), 3.78(\mathrm{~s}, 3 \mathrm{H})$, $3.41(\mathrm{ddd}, \mathrm{J}=10.4,5.8,3.3 \mathrm{~Hz}, 1 \mathrm{H}), 3.37-3.32(\mathrm{~m}, 2 \mathrm{H}), 3.29$ (s, 3H), 3.17 (ddd, J = 10.4, 5.2, $3.3 \mathrm{~Hz}, 1 \mathrm{H}), 2.50$ (ddd, J = 14.7, 6.1, $1.8 \mathrm{~Hz}, 1 \mathrm{H}), 2.34$ (ddd, J = 14.7, 6.0, $3.3 \mathrm{~Hz}, 1 \mathrm{H}$ ), $2.03(\mathrm{~s}, 3 \mathrm{H}),{ }^{13} \mathrm{C}\left\{{ }^{1} \mathrm{H}\right\}$ NMR $(100 \mathrm{MHz}, \mathrm{CDCl} 3) \delta: 170.2,152.8,137.3,131.1,104.1,97.4$, 93.9, 83.7, 76.5, 71.4, 66.7, 60.8, 58.8, 56.0, 40.3, 21.2; LRMS (ESI) $423.2[\mathrm{M}+\mathrm{Na}]^{+}$; HRMS (ESI-Orbitrap) m/z: [M+Na $]^{+}$calcd for $\mathrm{C}_{19} \mathrm{H}_{28} \mathrm{O}_{9} \mathrm{Na} 423.1626$, found 423.1623 .

\section{General procedure for the allylation of tetrahydrofuranyl acetate}

A solution of tetrahydrofuranyl acetate in distilled $\mathrm{CH}_{2} \mathrm{Cl}_{2}$ at $-78{ }^{\circ} \mathrm{C}$ under argon atmosphere was treated with allyltrimethylsilane (4.0 equiv) and Lewis acid. The resulting reaction mixture was stirred at $-78{ }^{\circ} \mathrm{C}$ for $3 \mathrm{~h}$. After this period, the reaction mixture was treated with saturated aqueous $\mathrm{Na}_{2} \mathrm{HPO}_{4}(1 \mathrm{~mL}$ per mmol of acetate). The aqueous layer was then extracted three times with $\mathrm{CH}_{2} \mathrm{Cl}_{2}$, and the collected organic phases were dried $\left(\mathrm{Na}_{2} \mathrm{SO}_{4}\right)$, filtered, and concentrated under reduced pressure.

\section{Preparation of (2R,3R,5S)-5-Allyl-3-(Methoxymethoxy)-2-(3,4,5-} Trimethoxyphenyl) Tetrahydrofuran (13a) and (2R,3R,5R)-5-Allyl-3(Methoxymethoxy)-2-(3,4,5-Trimethoxyphenyl)Tetrahydrofuran (14a) mixture.Following the general procedure described above and starting from acetate $\mathbf{6}(18 \mathrm{mg}, 0.05$ $\mathrm{mmol}$ ) in $\mathrm{CH}_{2} \mathrm{Cl}_{2}(1 \mathrm{~mL})$, compounds $13 \mathbf{a}$ and $14 \mathbf{a}(14.4 \mathrm{mg}, 84 \%$ yield) were obtained as a colorless oil after the crude residue was purified by column chromatography over silica gel (5-20\% EtOAc in hexanes). The results are summarized in Table S1. ${ }^{1} \mathrm{H}$ NMR (mixture, 400 $\left.\mathrm{MHz}, \mathrm{CDCl}_{3}\right)$ \&: $6.64(\mathrm{~s}, 3.2 \mathrm{H}), 6.61(\mathrm{~s}, 2 \mathrm{H}), 6.00-5.74(\mathrm{~m}, 2.6 \mathrm{H}), 5.18-5.04(\mathrm{~m}, 5.2 \mathrm{H})$, $4.93(\mathrm{~d}, \mathrm{~J}=3.3 \mathrm{~Hz}, 1 \mathrm{H}), 4.73(\mathrm{~d}, \mathrm{~J}=4.1 \mathrm{~Hz}, 1.6 \mathrm{H}), 4.58-4.44(\mathrm{~m}, 1 \mathrm{H}), 4.41-4.25(\mathrm{~m}$, $5.3 \mathrm{H}), 4.18-4.01(\mathrm{~m}, 4.8 \mathrm{H}), 3.85(\mathrm{~s}, 15.2 \mathrm{H}), 3.81(\mathrm{~s}, 4.6 \mathrm{H}), 3.81(\mathrm{~s}, 3 \mathrm{H}), 3.07(\mathrm{~s}, 4.8 \mathrm{H})$, $3.04(\mathrm{~s}, 3 \mathrm{H}), 2.70-2.57(\mathrm{~m}, 1.7 \mathrm{H}), 2.52-2.29(\mathrm{~m}, 5.8 \mathrm{H}), 2.19$ (ddd, 1H), 1.91 (ddd, J = $13.2 \mathrm{~Hz}, 1 \mathrm{H}), 1.85$ (ddd, $\mathrm{J}=11.4,6.0 \mathrm{~Hz}, 1.7 \mathrm{H}) ;{ }^{13} \mathrm{C}\left\{{ }^{1} \mathrm{H}\right\}$ NMR (mixture, $100 \mathrm{MHz}$, $\left.\mathrm{CDCl}_{3}\right)$ \&: 152.8, 134.8, 134.1, 133.6, 133.1, 117.4, 116.9, 104.2, 104.0, 95.0, 84.30, 83.7, 78.7, 77.8, 77.6, 77.1, 60.8, 56.0, 55.1, 40.4, 40.2, 38.8, 38.3; LRMS (ESI) m/z: 361.2 [M $+\mathrm{Na}]^{+}$; HRMS (ESI-Orbitrap) m/z: [M+Na] ${ }^{+}$calcd for $\mathrm{C}_{18} \mathrm{H}_{26} \mathrm{O}_{6} \mathrm{Na} 361.1622$, found 361.1624 .

\section{Preparation of (((2R,3R,5S)-5-Allyl-2-(3,4,5-} Trimethoxyphenyl)Tetrahydrofuran-3-yl)oxy)(tert-butyl)Dimethylsilane (13b).Following the general procedure described above and starting from acetate $\mathbf{1 1}(21.7 \mathrm{mg}, 0.05$ $\mathrm{mmol})$ in $\mathrm{CH}_{2} \mathrm{Cl}_{2}(1 \mathrm{~mL})$, the title compound $\mathbf{1 3 b}$ (16.8 $\mathrm{mg}, 81 \%$ yield) was prepared as a colorless oil after the crude residue was purified by column chromatography over silica gel (5-20\% EtOAc in hexanes). The results are summarized in Table S1. [a $]_{\mathrm{D}}{ }^{20}-62.0$ ( $c 0.79$, $\left.\mathrm{CHCl}_{3}\right) ;{ }^{1} \mathrm{H} \mathrm{NMR}\left(400 \mathrm{MHz}, \mathrm{CDCl}_{3}\right) \delta 6.59(\mathrm{~s}, 2 \mathrm{H}), 5.99-5.85(\mathrm{~m}, 1 \mathrm{H}), 5.18-5.04(\mathrm{~m}$, $2 \mathrm{H}), 4.73(\mathrm{~d}, \mathrm{~J}=3.7 \mathrm{~Hz}, 1 \mathrm{H}), 4.30(\mathrm{td}, \mathrm{J}=3.7,1.8 \mathrm{~Hz}, 1 \mathrm{H}), 4.22-4.13(\mathrm{~m}, 1 \mathrm{H}), 3.84(\mathrm{~s}$, 
6H), 3.79 (s, 3H), 2.67 (dt, J = 13.6, $6.7 \mathrm{~Hz}, 1 \mathrm{H}), 2.48(\mathrm{dt}, \mathrm{J}=13.8,6.9 \mathrm{~Hz}, 1 \mathrm{H}), 2.35$ (ddd, J $=13.6,8.5,5.4 \mathrm{~Hz}, 1 \mathrm{H}), 1.80(\mathrm{ddd}, \mathrm{J}=13.2,4.4,1.7 \mathrm{~Hz}, 1 \mathrm{H}), 0.68(\mathrm{~s}, 9 \mathrm{H}),-0.13(\mathrm{~s}, 3 \mathrm{H})$, $-0.37(\mathrm{~s}, 3 \mathrm{H}) ;{ }^{13} \mathrm{C}\left\{{ }^{1} \mathrm{H}\right\}$ NMR $\left(100 \mathrm{MHz}, \mathrm{CDCl}_{3}\right)$ 8: 152.6, 135.3, 133.9, 116.6, 104.6, 85.9, 77.4, 74.6, 60.7, 55.9, 41.0, 40.6, 25.5, 17.7, -5.3, -5.6; LRMS (ESI) $431.2[\mathrm{M}+\mathrm{Na}]^{+}$; HRMS (ESI-Orbitrap) m/z: [M+H] ${ }^{+}$calcd for $\mathrm{C}_{22} \mathrm{H}_{37} \mathrm{O}_{5} \mathrm{Si} 409.2405$, found 409.2401.

\section{Preparation of (((2R,3R,5S)-5-Allyl-2-(3,4,5-}

Trimethoxyphenyl)Tetrahydrofuran-3-yl)oxy)(tert-butyl)Dimethylsilane (13c).Following the general procedure described above and starting from acetate $12(20 \mathrm{mg}, 0.05$ $\mathrm{mmol})$ in $\mathrm{CH}_{2} \mathrm{Cl}_{2}(1 \mathrm{~mL})$, the title compound $\mathbf{1 3 c}(6.3 \mathrm{mg}, 33 \%$ yield) was prepared as a colorless oil after the crude residue was purified by column chromatography over silica gel (40\% EtOAc in hexanes). The results are summarized in Table S1. [a $]_{\mathrm{D}}{ }^{20}-85.0$ (c 0.12, $\left.\mathrm{CHCl}_{3}\right) ;{ }^{1} \mathrm{H} \mathrm{NMR}\left(800 \mathrm{MHz}, \mathrm{CDCl}_{3}\right) \delta 6.63(\mathrm{~s}, 2 \mathrm{H}), 5.91-5.86(\mathrm{~m}, 1 \mathrm{H}), 5.15(\mathrm{dd}, \mathrm{J}=$ $35.1,13.7 \mathrm{~Hz}, 2 \mathrm{H}), 4.96(\mathrm{~d}, \mathrm{~J}=3.2 \mathrm{~Hz}, 1 \mathrm{H}), 4.53(\mathrm{dq}, \mathrm{J}=12.2,6.1 \mathrm{~Hz}, 1 \mathrm{H}), 4.49(\mathrm{~d}, \mathrm{~J}=7.2$ $\mathrm{Hz}, 1 \mathrm{H}), 4.43(\mathrm{t}, \mathrm{J}=3.7 \mathrm{~Hz}, 1 \mathrm{H}), 4.28(\mathrm{~d}, \mathrm{~J}=7.2 \mathrm{~Hz}, 1 \mathrm{H}), 3.88(\mathrm{~s}, 6 \mathrm{H}), 3.84(\mathrm{~s}, 3 \mathrm{H}), 3.44$ (ddd, J = 10.7, 6.2, $3.2 \mathrm{~Hz}, 1 \mathrm{H}), 3.41-3.36(\mathrm{~m}, 2 \mathrm{H}), 3.35$ (s, 3H), 3.19 (ddd, J = 10.7, 5.5, $3.2 \mathrm{~Hz}, 1 \mathrm{H}), 2.51(\mathrm{dt}, \mathrm{J}=13.2,6.3 \mathrm{~Hz}, 1 \mathrm{H}), 2.41(\mathrm{dt}, \mathrm{J}=14.0,6.9 \mathrm{~Hz}, 1 \mathrm{H}), 2.25(\mathrm{dd}, \mathrm{J}=$ 13.3, $5.9 \mathrm{~Hz}, 1 \mathrm{H}), 1.93(\mathrm{ddd}, \mathrm{J}=13.6,9.6,4.5 \mathrm{~Hz}, 1 \mathrm{H}) ;{ }^{13} \mathrm{C}\left\{{ }^{1} \mathrm{H}\right\} \mathrm{NMR}\left(200 \mathrm{MHz}, \mathrm{CDCl}_{3}\right)$ \&: 152.9, 137.2, 134.2, 133.8, 117.5, 104.1, 94.0, 83.8, 78.7, 77.8, 71.6, 66.6, 60.9, 59.0, 56.1, 40.3, 38.8; LRMS (ESI) $405.1[\mathrm{M}+\mathrm{Na}]^{+}$; HRMS (ESI-Orbitrap) m/z: $[\mathrm{M}+\mathrm{Na}]^{+}$calcd for $\mathrm{C}_{20} \mathrm{H}_{30} \mathrm{O}_{7} \mathrm{Na} 405.1884$, found 405.1882 .

(2S,3aR,9bR)-2-Allyl-6,7,8-Trimethoxy-3,3a,5,9b-Tetrahydro-2H-furo[3,2c]lsochromene (17).-To a stirred solution of acetate $\mathbf{6}(13.2 \mathrm{mg}, 0.037 \mathrm{mmol})$ in $\mathrm{CH}_{2} \mathrm{Cl}_{2}(2.4 \mathrm{~mL})$ at $-78{ }^{\circ} \mathrm{C}$ under argon atmosphere were added allyltrimethylsilane $(24 \mu \mathrm{L}$, $0.148 \mathrm{mmol}$ ) and $\mathrm{SnBr}_{4}(26 \mathrm{mg}, 0.059 \mathrm{mmol}, 1.6$ equiv). The reaction mixture was stirred for $3 \mathrm{~h}$ at the same temperature and the reaction progress was monitored by TLC. When the starting material was completely consumed, additional portion of $\mathrm{SnBr}_{4}(33 \mathrm{mg}, 0.075$ mmol, 2.0 equiv) was added at $0{ }^{\circ} \mathrm{C}$ and the resulting mixture was warmed to $23{ }^{\circ} \mathrm{C}$ overnight. After this period, the reaction mixture was quenched by the addition of saturated aqueous $\mathrm{Na}_{2} \mathrm{HPO}_{4}(0.4 \mathrm{~mL})$ and extracted with dichloromethane (2x). The extracts were dried over $\mathrm{Na}_{2} \mathrm{SO}_{4}$, filtered, and concentrated under reduced pressure. NMR analysis of the unpurified crude product showed a pair of diastereomers in a 4:1 ratio (cis:trans diastereomers). The crude product was purified by column chromatography over silica gel (10\% EtOAc in hexanes) to give $\mathbf{1 7}$ and $\mathbf{1 8}(5.9 \mathrm{mg}, 52 \%)$ as a colorless oil. [a $]_{\mathrm{D}}{ }^{20}+25.6(c$ 0.67, $\mathrm{CHCl}_{3}$ ); cis-isomer 17 (major): ${ }^{1} \mathrm{H} \mathrm{NMR}\left(400 \mathrm{MHz}, \mathrm{CDCl}_{3}\right) \delta: 6.79(\mathrm{~s}, 1 \mathrm{H}), 5.96-$ $5.64(\mathrm{~m}, 1 \mathrm{H}), 5.21-4.97(\mathrm{~m}, 2 \mathrm{H}), 4.90(\mathrm{~d}, J=15.1 \mathrm{~Hz}, 1 \mathrm{H}), 4.42(\mathrm{~d}, J=15.1 \mathrm{~Hz}, 1 \mathrm{H}), 4.30$ (d, $J=3.3 \mathrm{~Hz}, 1 \mathrm{H}), 4.16(\mathrm{ddd}, J=6.8,3.3,1.7 \mathrm{~Hz}, 1 \mathrm{H}), 4.02(\mathrm{qd}, J=7.4,5.8 \mathrm{~Hz}, 1 \mathrm{H}), 3.87$ (s, 3H), 3.86 (s, 3H), 3.84 (s, 3H), $2.65-2.44(\mathrm{~m}, 2 \mathrm{H}), 2.43-2.29$ (m, 1H), 1.81 (ddd, $J=$ 14.0, 7.2, $1.7 \mathrm{~Hz}, 1 \mathrm{H}) ;{ }^{13} \mathrm{C}\left\{{ }^{1} \mathrm{H}\right\} \mathrm{NMR}\left(100 \mathrm{MHz}, \mathrm{CDCl}_{3}\right)$ 8: 152.9, 149.0, 141.7, 134.7, 126.5, 121.8, 117.1, 108.7, 78.1, 75.4, 63.3, 60.9, 60.7, 56.1, 40.3, 39.0; LRMS (ESI) 307.1 $[\mathrm{M}+\mathrm{H}]^{+}$; HRMS (ESI-Orbitrap) m/z: [M+Na $]^{+}$calcd for $\mathrm{C}_{17} \mathrm{H}_{22} \mathrm{O}_{5} 329.1359$, found 329.1357; Trans-isomer 18 (minor): ${ }^{1} \mathrm{H}$ NMR (400 MHz, $\mathrm{CDCl}_{3}$ ) $\delta: 6.79$ (s, 1H), 5.92 $5.76(\mathrm{~m}, 1 \mathrm{H}), 5.18-5.04(\mathrm{~m}, 2 \mathrm{H}), 4.91(\mathrm{~d}, \mathrm{~J}=15.0 \mathrm{~Hz}, 1 \mathrm{H}), 4.61(\mathrm{~d}, \mathrm{~J}=3.0 \mathrm{~Hz}, 1 \mathrm{H}), 4.45-$ $4.32(\mathrm{~m}, 2 \mathrm{H}), 4.26-4.18(\mathrm{~m}, 1 \mathrm{H}), 3.88(\mathrm{~s}, 3 \mathrm{H}), 3.86(\mathrm{~s}, 3 \mathrm{H}), 3.84(\mathrm{~s}, 3 \mathrm{H}), 2.48(\mathrm{dt}, \mathrm{J}=12.6$, 
$6.2 \mathrm{~Hz}, 1 \mathrm{H}), 2.34(\mathrm{dt}, \mathrm{J}=14.0,7.0 \mathrm{~Hz}, 1 \mathrm{H}), 2.26(\mathrm{dd}, \mathrm{J}=13.5,5.7 \mathrm{~Hz}, 1 \mathrm{H}), 1.94$ (ddd, J =

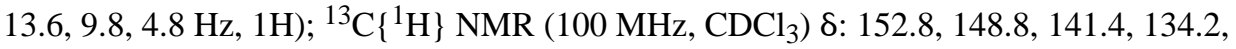
127.2, 121.0, 117.3, 108.4, 77.6, 77.4, 74.5, 62.8, 60.7, 60.6, 56.0, 39.9, 39.4; LRMS (ESI) $307.1[\mathrm{M}+\mathrm{H}]^{+}$.

(2S,3aR,9bR)-6,7,8-Trimethoxy-2-Propyl-3,3a,5,9b-Tetrahydro-2H-furo[3,2c]lsochromene (19).-To a stirred of isochromene 17 (47.4 mg, $0.156 \mathrm{mmol})$ in EtOAc $(2 \mathrm{~mL})$ at $23{ }^{\circ} \mathrm{C}$ was added $10 \% \mathrm{Pd} / \mathrm{C}(10 \mathrm{mg})$. The resulting solution was stirred at $23{ }^{\circ} \mathrm{C}$ under a hydrogen filled balloon over $12 \mathrm{~h}$. Upon completion, the mixture was filtered through a plug of Celite and solvents were removed under reduced pressure. The crude product was purified by silica gel column chromatography (20\% EtOAc in hexane) to give propyl derivative $19(42.2 \mathrm{mg}, 88 \%)$. $[a]_{\mathrm{D}}^{20}+15.3\left(c 0.40, \mathrm{CHCl}_{3}\right)$; reported $[\mathrm{a}]_{\mathrm{D}}{ }^{26}+16.5$ $\left(c 1.02, \mathrm{CHCl}_{3}\right)^{17} ;{ }^{1} \mathrm{H} \mathrm{NMR}\left(400 \mathrm{MHz}, \mathrm{CDCl}_{3}\right) \delta: 6.80(\mathrm{~s}, 1 \mathrm{H}), 4.89(\mathrm{~d}, J=15.0 \mathrm{~Hz}, 1 \mathrm{H})$, $4.41(\mathrm{~d}, J=15.1 \mathrm{~Hz}, 1 \mathrm{H}), 4.26$ (d, $J=3.3 \mathrm{~Hz}, 1 \mathrm{H}), 4.20-4.09$ (m, $1 \mathrm{H}), 3.93$ (q, $J=7.1 \mathrm{~Hz}$, $1 \mathrm{H}), 3.87$ (s, 3H), $3.86(\mathrm{~s}, 3 \mathrm{H}), 3.84(\mathrm{~s}, 3 \mathrm{H}), 2.52(\mathrm{dt}, J=14.3,7.3 \mathrm{~Hz}, 1 \mathrm{H}), 1.80-1.69(\mathrm{~m}$, $2 \mathrm{H}), 1.61-1.52(\mathrm{~m}, 1 \mathrm{H}), 1.47-1.36(\mathrm{~m}, 2 \mathrm{H}), 0.92(\mathrm{t}, J=7.3 \mathrm{~Hz}, 3 \mathrm{H}) ;{ }^{13} \mathrm{C}\left\{{ }^{1} \mathrm{H}\right\} \mathrm{NMR}(100$ $\left.\mathrm{MHz}, \mathrm{CDCl}_{3}\right)$ \&: 152.8, 148.9, 141.6, 126.6, 121.8, 108.7, 78.6, 77.4, 75.2, 63.3, 60.9, 60.7, 56.1, 39.6, 38.1, 19.6, 14.1; LRMS (ESI) $309.1[\mathrm{M}+\mathrm{H}]^{+}$.

(2S,3aR,9bR)-6,7,8-Trimethoxy-2-Propyl-2,3,3a,9b-Tetrahydro-5H-furo[3,2c]lsochromen-5-one (20).-To a solution of propyl derivative $19(41.2 \mathrm{mg}, 0.135 \mathrm{mmol})$ in $\mathrm{CH}_{2} \mathrm{Cl}_{2}(4 \mathrm{~mL})$, pyridine ( $\left.54 \mu \mathrm{L}, 0.67 \mathrm{mmol}\right)$ and $\mathrm{CrO}_{3}(40 \mathrm{mg}, 0.40 \mathrm{mmol})$ were added and stirred for $36 \mathrm{~h}$ at $23{ }^{\circ} \mathrm{C}$. After this period, the reaction mixture was concentrated under reduced pressure and ethyl acetate $(10 \mathrm{ml})$ was added and filtered. The filtrate was washed with aq. $\mathrm{CuSO}_{4}$ solution followed by water. The organic phase was dried over $\mathrm{Na}_{2} \mathrm{SO}_{4}$, filtered, and concentrated under reduced pressure. Flash chromatography over silica gel (30\% EtOAc in hexanes) provided $\delta$-valerolactone $\mathbf{2 0}(17.2 \mathrm{mg}, 40 \% ; 60 \% \mathrm{brsm})$ as a paleyellow oil with the recovered starting material $19(13.7 \mathrm{mg})$. [a $]_{\mathrm{D}}{ }^{20}+20.4\left(c 0.31, \mathrm{CHCl}_{3}\right)$; reported ${ }^{18}[\mathrm{a}]_{\mathrm{D}}{ }^{26}+21.5\left(c 0.50, \mathrm{CHCl}_{3}\right) ;{ }^{1} \mathrm{H} \mathrm{NMR}\left(400 \mathrm{MHz}, \mathrm{CDCl}_{3}\right) \delta: 6.78(\mathrm{~s}, 1 \mathrm{H}), 4.94$ (ddd, $J=5.8,2.9,1.0 \mathrm{~Hz}, 1 \mathrm{H}), 4.51(\mathrm{~d}, J=2.9 \mathrm{~Hz}, 1 \mathrm{H}), 4.25-4.06(\mathrm{~m}, 1 \mathrm{H}), 3.97(\mathrm{~s}, 3 \mathrm{H})$, $3.94(\mathrm{~s}, 3 \mathrm{H}), 3.88(\mathrm{~s}, 3 \mathrm{H}), 2.51$ (ddd, $J=14.4,8.8,5.8 \mathrm{~Hz}, 1 \mathrm{H}), 2.16$ (ddd, $J=14.3,5.5,1.1$ $\mathrm{Hz}, 1 \mathrm{H}), 1.76-1.69(\mathrm{~m}, 1 \mathrm{H}), 1.66-1.58(\mathrm{~m}, 1 \mathrm{H}), 1.45-1.31(\mathrm{~m}, 2 \mathrm{H}), 0.91(\mathrm{t}, J=7.4 \mathrm{~Hz}$, $3 \mathrm{H}) ;{ }^{13} \mathrm{C}\left\{{ }^{1} \mathrm{H}\right\} \mathrm{NMR}\left(100 \mathrm{MHz}, \mathrm{CDCl}_{3}\right) \delta: 159.8,157.9,156.4,144.2,132.5,111.2,108.0$, 79.4, 78.9, 75.0, 61.7, 61.0, 56.1, 39.0, 38.0, 19.1, 13.9; LRMS (ESI) $323.0[\mathrm{M}+\mathrm{H}]^{+}$; HRMS (ESI-Orbitrap) m/z: [M+Na] $]^{+}$calc'd for $\mathrm{C}_{17} \mathrm{H}_{22} \mathrm{O}_{6} \mathrm{Na} 345.1309$, found 345.1306.

(+)-Monocerin (1).-To a solution of $\delta$-valerolactone 20 (13.4 mg, $0.042 \mathrm{mmol})$ in dry $\mathrm{CH}_{2} \mathrm{Cl}_{2}(1 \mathrm{~mL})$ at $-10{ }^{\circ} \mathrm{C}$ under argon atmosphere was added $\mathrm{BCl}_{3}(1.0 \mathrm{M}$ in DCM, $50 \mu \mathrm{L}$, $0.11 \mathrm{mmol})$. The mixture was stirred at $-10^{\circ} \mathrm{C}$ for $2 \mathrm{~h}$ and the reaction was quenched by the addition of saturated aqueous $\mathrm{NaHCO}_{3}(1 \mathrm{~mL})$. The mixture was extracted with ethyl acetate and the organic layer was washed with brine, dried over $\mathrm{Na}_{2} \mathrm{SO}_{4}$, filtered, and concentrated under reduced pressure. Purification by column chromatography over silica gel (20\% EtOAc in hexanes) gave (+)-monocerin (1) (4.9 mg, $41 \%$ yield; $88 \%$ yield brsm) as a colorless oil with the recovered starting material $20(7.6 \mathrm{mg})$. $[\mathrm{a}]_{\mathrm{D}}{ }^{20}+57.8\left(c 0.29, \mathrm{CHCl}_{3}\right)$; reported ${ }^{6}$ $[\mathrm{a}]_{\mathrm{D}}{ }^{24}+53.0($ c $0.85, \mathrm{MeOH}) ;{ }^{1} \mathrm{H} \mathrm{NMR}\left(400 \mathrm{MHz}, \mathrm{CDCl}_{3}\right) \delta: 11.28(\mathrm{~s}, 1 \mathrm{H}), 6.59(\mathrm{~s}, 1 \mathrm{H})$, 
5.05 (ddd, $J=6.3,3.2,1.2 \mathrm{~Hz}, 1 \mathrm{H}), 4.54(\mathrm{~d}, \mathrm{~J}=3.1 \mathrm{~Hz}, 1 \mathrm{H}), 4.12(\mathrm{dq}, J=8.6,6.3 \mathrm{~Hz}, 1 \mathrm{H})$, 3.95 (s, 3H), 3.89 (s, 3H), 2.59 (ddd, $J=14.6,8.6,6.2 \mathrm{~Hz}, 1 \mathrm{H}), 2.16$ (ddd, $J=14.5,5.9,1.2$ $\mathrm{Hz}, 1 \mathrm{H}), 1.74-1.65(\mathrm{~m}, 1 \mathrm{H}), 1.62-1.52(\mathrm{~m}, 1 \mathrm{H}), 1.49-1.29(\mathrm{~m}, 2 \mathrm{H}), 0.91(\mathrm{t}, J=7.3 \mathrm{~Hz}$, $3 \mathrm{H}) ;{ }^{13} \mathrm{C}\left\{{ }^{1} \mathrm{H}\right\}$ NMR $\left(100 \mathrm{MHz}, \mathrm{CDCl}_{3}\right) \delta: 167.9,158.8,156.4,137.5,131.3,104.5,102.2$, 81.4, 78.9, 74.6, 60.9, 56.4, 39.2, 38.2, 19.3, 14.1; LRMS (ESI) $\left.309.1[\mathrm{M}+\mathrm{H}]^{+}\right)$HRMS (ESI-Orbitrap) $\mathrm{m} / \mathrm{z}$ : $[\mathrm{M}+\mathrm{H}]^{+}$calcd for $\mathrm{C}_{16} \mathrm{H}_{21} \mathrm{O}_{6} 309.1333$, found 309.1335 .

(+)-Acetylmonocerin (2).-Acetic anhydride $(75 \mu \mathrm{L}, 0.8 \mathrm{mmol})$ and DMAP (catalytic amount) were added to a solution of (+)-monocerin (1) $(2.2 \mathrm{mg}, 0.007 \mathrm{mmol})$ in $0.2 \mathrm{~mL}$ of distilled pyridine at $0{ }^{\circ} \mathrm{C}$ under argon atmosphere, and the mixture was stirred at $23{ }^{\circ} \mathrm{C}$ for 5 h. After removing the solvent under reduced pressure, the crude product was purified by flash column chromatography on silica gel (33\% EtOAc in hexanes) to give (+)acetylmonocerin (2) $(2.4 \mathrm{mg}, 96 \%$ yield $)$ as a white amorphous solid. $[\mathrm{a}]_{\mathrm{D}}{ }^{25}+3.6(c 0.29$, $\left.\mathrm{CHCl}_{3}\right)$; reported ${ }^{19}[\mathrm{a}]_{\mathrm{D}}{ }^{24}-3.0(c 0.1, \mathrm{EtOH})$ for enantiomer of compound $2 ;{ }^{1} \mathrm{H} \mathrm{NMR}$ $\left(800 \mathrm{MHz}, \mathrm{CDCl}_{3}\right) \delta: 6.90(\mathrm{~s}, 1 \mathrm{H}), 4.99(\mathrm{~m}, 1 \mathrm{H}), 4.55(\mathrm{~d}, \mathrm{~J}=3.1 \mathrm{~Hz}, 1 \mathrm{H}), 4.14(\mathrm{~m}, 1 \mathrm{H})$, 3.97 (s, 3H), 3.85 (s, 3H), $2.53(\mathrm{ddd}, \mathrm{J}=14.5,8.7,5.9 \mathrm{~Hz}, 1 \mathrm{H}), 2.42$ (s, 3H), 2.13 (dd, J = $14.3,5.5 \mathrm{~Hz}, 1 \mathrm{H}), 1.71-1.64(\mathrm{~m}, 1 \mathrm{H}), 1.58-1.54(\mathrm{~m}, 1 \mathrm{H}), 1.44-1.39(\mathrm{~m}, 1 \mathrm{H}), 1.35-$ $1.32(\mathrm{~m}, 1 \mathrm{H}), 0.90(\mathrm{t}, \mathrm{J}=7.3 \mathrm{~Hz}, 3 \mathrm{H}) ;{ }^{13} \mathrm{C}\left\{{ }^{1} \mathrm{H}\right\} \mathrm{NMR}\left(200 \mathrm{MHz}, \mathrm{CDCl}_{3}\right)$ 8: 169.3, 159.9, 158.0, 146.1, 143.3, 132.7, 110.4, 109.9, 79.9, 79.0, 74.6, 61.2, 56.3, 39.0, 38.2, 21.0, 19.1, 14.0; LRMS (ESI) $373.1[\mathrm{M}+\mathrm{Na}]^{+}$.

\section{Supplementary Material}

Refer to Web version on PubMed Central for supplementary material.

\section{ACKNOWLEDGEMENTS}

Financial support of this work was provided by the National Institutes of Health (GM122279) and Purdue University.

\section{REFERENCES}

(1). Hill RA, Naturally occuring isocoumarines In: Progress in the Chemistry of Organic Natural Products, Wien, New York, Springer Verlag 1986, Vol 49, 1-78.

(2). Pal S; Chatare V; Pal M Isocoumarin and Its Derivatives: An Overview on their Synthesis and Applications. Curr. Org. Chem, 2011, 15, 782-800.

(3). Markaryan EA; Samodurova AG Advances in the Chemistry of Isochroman. Russ. Chem. Rev, 1989, 58, 479-493.

(4). Huang Y-F; Li L-H; Tian L; Qiao L; Hua H-M; Pei Y-H Sg17-1-4, a Novel Isocoumarin from a Marine Fungus Alternaria tenuis Sg17-1. J. Antibiot, 2006, 59, 355-357. [PubMed: 16915820]

(5). Ortiz A; Castro M; Sansinenea E 3,4-Dihydroisocoumarins, Interesting Natural Products: Isolation, Organic Syntheses and Biological Activities. Curr. Org. Chem, 2018, DOI: 10.2174/1570179415666180924123439.

(6). Aldridge DC; Turner WB Metabolites of Helminthosporium monoceras: structures of monocerin and related benzopyrans. J. Chem. Soc., C, 1970, 2598-2600.

(7). Grove JF; Pople MJ Metabolic products of Fusarium larvarum fuckel. The fusarentins and the absolute configuration of monocerin. J. Chem. Soc., Perkin Trans. 1, 1979, 2048-2051.

(8). Scott FE; Simpson TJ; Trimble LA; Vederas JC Biosynthesis of Monocerin. Incorporation of ${ }^{2} \mathrm{H}-$, ${ }^{13} \mathrm{C}-$, and ${ }^{18} \mathrm{O}$-labelled acetates by Drechslera ravenelii. J. Chem. Soc., Chem. Commun, 1984, $756-758$. 
(9). Axford LC; Simpson TJ; Willis CL; Synthesis and Biosynthesis of the First PKS-free Intermediate in Monocerin Biosynthesis. Angew. Chem. Int. Ed. 43, 2004, 727-730.

(10). Cuq F; Petitprez M; Herrmann-Gorline S; Klaebe A; Rossignol M Monocerin in Exserohilum turcicum isolates from maize and a study of its phytotoxicity. Phytochemistry, 1993, 34, 12651270.

(11). Zhang W; Krohn K; Draeger S; Schulz B Bioactive Isocoumarins Isolated from the Endophytic Fungus Microdochium bolleyi. J. Nat. Prod, 2008, 71, 1078-1081. [PubMed: 18510362]

(12). Robeson DJ; Strobel GA Monocerin, a Phytotoxin from Exserohilum turcicum (三 Drechslera turcica). Agric. \& Biol. Chem, 1982, 46, 2681-2683.

(13). Haritakun R; Sappan M; Suvannakad R; Tasanathai K; Isaka M An Antimycobacterial Cyclodepsipeptide from the Entomopathogenic Fungus Ophiocordyceps communis BCC 16475. J. Nat. Prod, 2010, 73, 75-78. [PubMed: 20028029]

(14). Sappapan R; Sommit D; Ngamrojanavanich N; Pengpreecha S; Wiyakrutta S; Sriubolmas N; Pudhom K 11-Hydroxymonocerin from the Plant Endophytic Fungus Exserohilum rostratum. J. Nat. Prod, 2008, 71, 1657-1659. [PubMed: 18774863]

(15). El Aouad N; Pérez-Moreno G; Sánchez P; Cantizani J; Ortiz-López FJ; Martín J; GonzálezMenéndez V; Ruiz-Pérez LM; González-Pacanowska D; Vicente F; Bills G; Reyes F Lasionectrin, a Naphthopyrone from a Lasionectria sp. J. Nat. Prod, 2012, 75, 1228-1230. [PubMed: 22694295]

(16). Mori K; Takaishi H Synthesis of monocerin, an antifungal, insecticidal and phytotoxic heptaketide metabolite of Exserohilum monoceras. Tetrahedron, 1989, 45, 1639-1646.

(17). Dillon MP; Simpson TJ; Sweeney JB Enantioselective synthesis of monocerin and fusarentin ethers: Antifungal and insecticidal fungal metabolites. Tetrahedron Lett, 1992, 33, 7569-7572.

(18). Fang B; Xie X; Zhao C; Jing P; Li H; Wang Z; Gu J; She X Asymmetric Total Synthesis of Fusarentin 6-Methyl Ether and Its Biomimetic Transformation into Fusarentin 6,7-Dimethyl Ether, 7-O-Demethylmonocerin, and (+)-Monocerin. J. Org. Chem, 2013, 78, 6338-6343. [PubMed: 23713975]

(19). Kwon HK; Lee YE; Lee E Radical Cyclization of Vinylic Ethers: Expedient Synthesis of (+)Monocerin. Org. Lett, 2008, 10, 2995-2996. [PubMed: 18549222]

(20). Cassidy JH; Farthing CN; Marsden SP; Pedersen A; Slater M; Stemp G A concise, convergent total synthesis of monocerin. Org. Biomol. Chem, 2006, 4, 4118-4126. [PubMed: 17312966]

(21). Fang B; Xie X; Li H; Jing P; Gu J; She X Asymmetric total synthesis of (+)-monocerin. Tetrahedron Lett, 2013, 54, 6349-6351.

(22). Fujita M; Mori K; Shimogaki M; Sugimura T Asymmetric Synthesis of 4,8Dihydroxyisochroman-1-one Polyketide Metabolites Using Chiral Hypervalent Iodine(III). Org. Lett, 2012, 14, 1294-1297. [PubMed: 22335341]

(23). Nookaraju U; Begari E; Kumar P Total synthesis of (+)-monocerin via tandem dihydroxylationSN2 cyclization and a copper mediated tandem cyanation-lactonization approach. Org. \& Biomol. Chem, 2014, 12, 5973-5980. [PubMed: 24988536]

(24). Poral VL; Furkert DP; Brimble MA Total Synthesis and Structural Confirmation of the Antimalarial Naphthopyrone Lasionectrin. Org. Lett, 2015, 17, 6214-6217. [PubMed: 26650711]

(25). Larsen CH; Ridgway BH; Shaw JT; Woerpel KA A Stereoelectronic Model To Explain the Highly Stereoselective Reactions of Nucleophiles with Five-Membered-Ring Oxocarbenium Ions. J. Am. Chem. Soc, 1999, 121, 12208-12209.

(26). Larsen CH; Ridgway BH; Shaw JT; Smith DM; Woerpel KA Stereoselective C-Glycosylation Reactions of Ribose Derivatives: Electronic Effects of Five-Membered Ring Oxocarbenium Ions. J. Am. Chem. Soc, 2005, 127, 10879-10884. [PubMed: 16076193]

(27). Kolb HC; VanNieuwenhze MS; Sharpless KB Catalytic Asymmetric Dihydroxylation. Chem. Rev, 1994, 94, 2483-2547.

(28). Sharpless KB; Amberg W; Bennani YL; Crispino GA; Hartung J; Jeong KS; Kwong HL; Morikawa K; Wang ZM; Xu D; Zhang XL The osmium-catalyzed asymmetric dihydroxylation: a new ligand class and a process improvement. J. Org. Chem, 1992, 57, 2768-2771. 
(29). Reitz AB; Mutter MS; Maryanoff BE Observation of cis and trans oxaphosphetanes in the Wittig reaction by high-field phosphorus-31 NMR spectroscopy. J. Am. Chem. Soc, 1984, 106, 18731875.

(30). Schlosser M; Christmann KF Olefinierungen mit Phosphor-Yliden, I. Mechanismus und Stereochemie der Wittig-Reaktion. Liebigs Ann. Chem, 1967, 708, 1-35.

(31). Reitz AB; Maryanoff BE Reversibility of a witting intermediate derived from a triphenylphosphonium ylide and an aliphatic aldehyde. J. Chem. Soc., Chem. Commun, 1984, 0, 1548-1549.

(32). Ghosh AK; Nyalapatla PR Enantioselective Total Synthesis of (+)-Amphirionin-4. Org. Lett, 2016, 18, 2296-2299. [PubMed: 27116318]

(33). Ghosh AK; Nyalapatla PR Total syntheses of both enantiomers of amphirionin 4: A chemoenzymatic based strategy for functionalized tetrahydrofurans. Tetrahedron, 2017, 73, 1820-1830. [PubMed: 28970641]

(34). Jung ME; Mossman AB; Lyster MA Direct synthesis of dibenzocyclooctadienes via double ortho Friedel-Crafts alkylation by the use of aldehyde-trimethylsilyl iodide adducts. J. Org. Chem, 1978, 43, 3698-3701.

(35). Larghi EL; Kaufman TS The Oxa-Pictet-Spengler Cyclization: Synthesis of Isochromans and Related Pyran-Type Heterocycles. Synthesis, 2006, 2006, 187-220. 


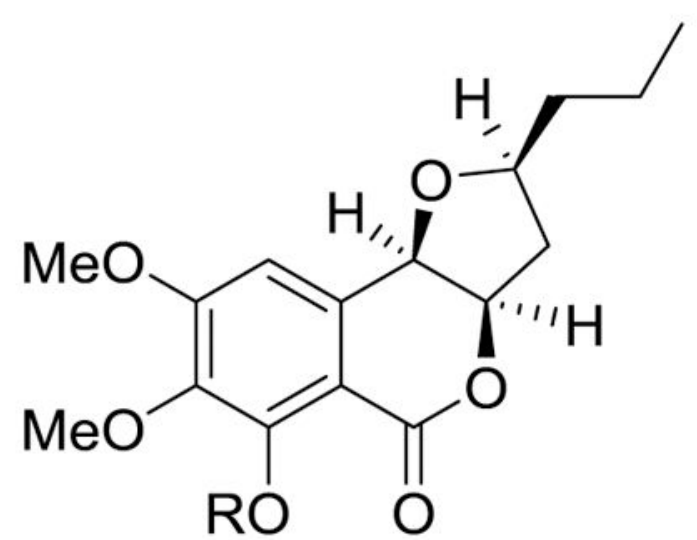

Monocerin, $1(\mathrm{R}=\mathrm{H})$

Acetoxy Monocerin $2(R=A c)$<smiles>COc1cc2c(c(O)c1OC)C(=O)O[C@H]1C[C@H](C[C@H](C)O)O[C@H]21</smiles>

11- $\beta$-Hydroxy Monocerin, 4

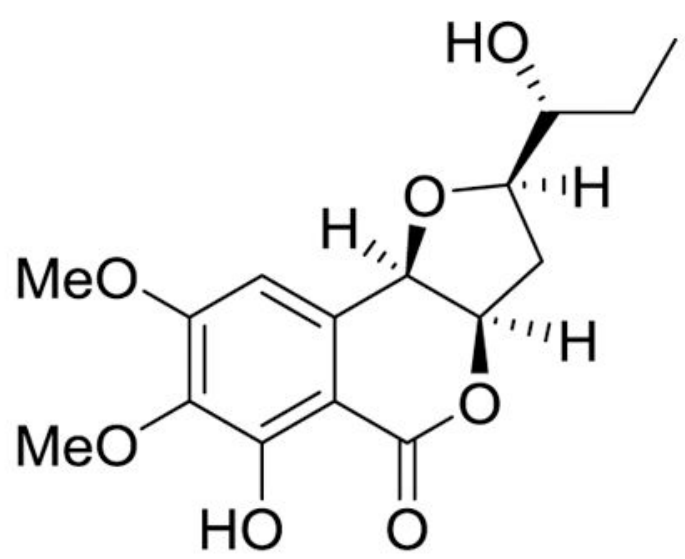

11- $\alpha$-Hydroxy Monocerin, 3

Figure 1.

Structures of dihydroisocoumarin natural products 1-5. 


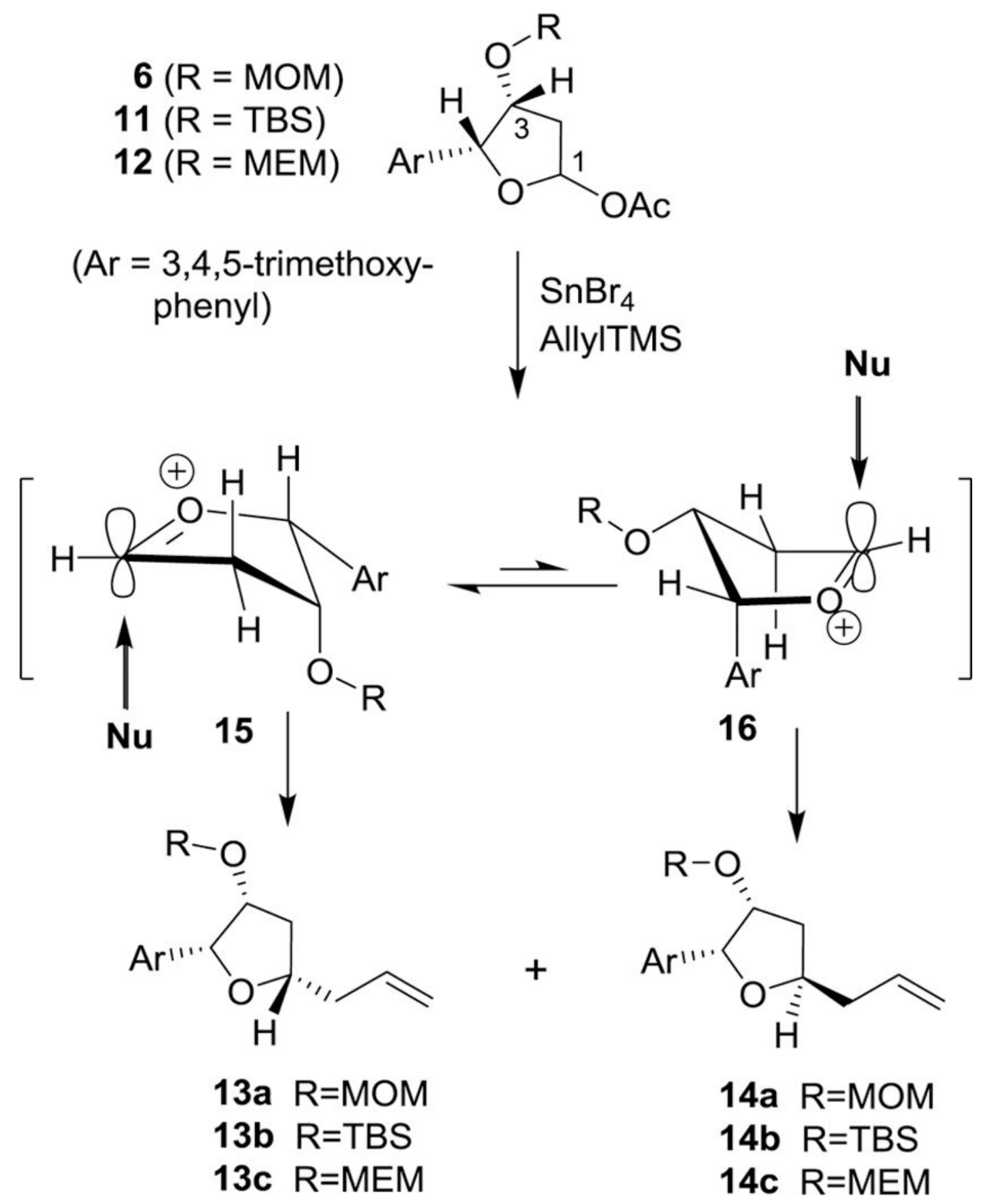

Figure 2.

Stereochemical analysis of cis-allylation reaction. 
<smiles>CCC[C@H]1C[C@H]2OC(=O)c3c(cc(OC)c(OC)c3O)[C@H]2O1</smiles>

1<smiles>COC(=O)C/C=C/c1cc(OC)c(OC)c(OC)c1</smiles><smiles>COc1cc([C@@H]2OC(=O)C[C@H]2O)cc(OC)c1OC</smiles>

Scheme 1.

Retrosynthetic analysis of (+)-monocerin 
<smiles>COC(=O)C/C=C/c1cc(OC)c(OC)c(OC)c1</smiles><smiles>COc1cc([C@@H]2OC(=O)C[C@H]2OC)cc(OC)c1OC</smiles><smiles>COc1cc([C@@H]2OC(=O)C[C@H]2O)cc(OC)c1OC</smiles><smiles>COc1cc([C@@H]2O[C@H](OC(C)=O)C[C@H]2OC)cc(OC)c1OC</smiles>

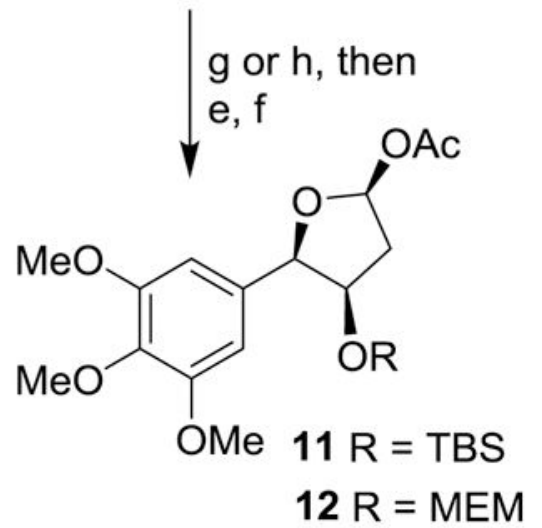<smiles>C=CC[C@H]1C[C@@H](OC)[C@H](c2cc(OC)c(OC)c(OC)c2)O1</smiles><smiles>C=CC[C@H]1C[C@@H](OC)[C@H](c2cc(OC)c(OC)c(OC)c2)O1</smiles>

Scheme 2.

Synthesis of allyl derivatives 13a and 14a. Reagents and Conditions: (a) $\mathrm{Ph}_{3} \mathrm{P}$ ${ }^{+} \mathrm{CH}_{2} \mathrm{CO}_{2} \mathrm{HBr}^{-}, t$-BuOK, THF, $-78{ }^{\circ} \mathrm{C}$ to $23{ }^{\circ} \mathrm{C}, 18 \mathrm{~h},(65 \%)$; (b) TMSCI, dry $\mathrm{MeOH}, 0{ }^{\circ} \mathrm{C}$ to $23{ }^{\circ} \mathrm{C}, 18 \mathrm{~h}$ (76\%); (c) $\mathrm{AD}-\mathrm{mix}-\beta, \mathrm{NaHCO}_{3}, \mathrm{MeSO}_{2} \mathrm{NH}_{2}, t-\mathrm{BuOH} / \mathrm{H}_{2} \mathrm{O}=1.1,0{ }^{\circ} \mathrm{C}, 24 \mathrm{~h}$ (90\%); (d) DIPEA, MOMCI, TBAI (cat.), THF, $50{ }^{\circ} \mathrm{C}, 24 \mathrm{~h}$ (95\%); (e) DIBAL-H, Toluene, $-78{ }^{\circ} \mathrm{C}$; (f) $\mathrm{Et}_{3} \mathrm{~N}$, DMAP, $\mathrm{Ac}_{2} \mathrm{O}, \mathrm{DCM}, 0{ }^{\circ} \mathrm{C}$ to $23{ }^{\circ} \mathrm{C}, 1.5 \mathrm{~h}$ (88\%); (g) TBSOTf, 2,6lutidine, DCM, $0{ }^{\circ} \mathrm{C}$ to $23{ }^{\circ} \mathrm{C}, 3 \mathrm{~h}(51 \%)$; (h) MEMCl, DIPEA, TBAI, THF, $0{ }^{\circ} \mathrm{C}$ to $55^{\circ} \mathrm{C}$, $72 \mathrm{~h}$ (79\%); (i) allyltrimethylsilane, $\mathrm{SnBr}_{4}, \mathrm{DCM},-78{ }^{\circ} \mathrm{C}, 3 \mathrm{~h},(84 \%)$. 
<smiles>COc1cc([C@@H]2O[C@H](OC(C)=O)C[C@H]2OC)cc(OC)c1OC</smiles><smiles>[CH]C</smiles><smiles>C=CC[C@H]1C[C@H]2OCc3c(cc(OC)c(OC)c3OC)[C@@H]2O1</smiles><smiles>CCC[C@H]1C[C@H]2OCc3c(cc(OC)c(OC)c3OC)[C@@H]2O1</smiles>

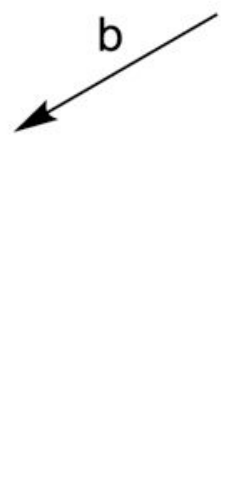<smiles>C=CC[C@H]1C[C@H]2OCc3c(cc(OC)c(OC)c3OC)[C@@H]2O1</smiles><smiles>CCC[C@H]1C[C@@H]2OC(=O)c3c(cc(OC)c(OC)c3OC)[C@@H]2O1</smiles>

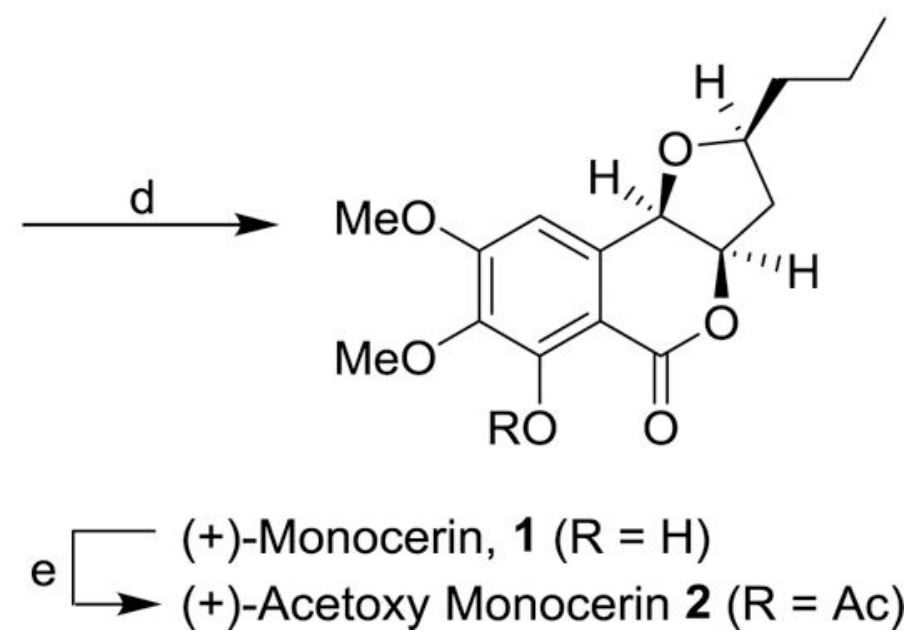

Scheme 3.

Synthesis of (+)-monocerin. Reagents and Conditions: (a) $\mathrm{SnBr}_{4}$, allyltrimethylsilane, $\mathrm{CH}_{2} \mathrm{Cl}_{2},-78{ }^{\circ} \mathrm{C}$ to $23^{\circ} \mathrm{C}, 18 \mathrm{~h}$ (52\%); (b) $\mathrm{H}_{2}, 10 \%$, Pd-C, EtOAc, $23{ }^{\circ} \mathrm{C}, 12 \mathrm{~h}(88 \%$ ); (c) $\mathrm{CrO}_{3}$, Pyridine, $\mathrm{CH}_{2} \mathrm{Cl}_{2}, 0{ }^{\circ} \mathrm{C}$ to $23{ }^{\circ} \mathrm{C}, 36 \mathrm{~h}$ (40\%, 60\% brsm); (d) $\mathrm{BCl}_{3}, \mathrm{CH}_{2} \mathrm{Cl}_{2},-10{ }^{\circ} \mathrm{C}, 2$ h $\left(41 \%, 88 \%\right.$ brsm); (e) $\mathrm{Ac}_{2} \mathrm{O}$, Pyridine, $\mathrm{CH}_{2} \mathrm{Cl}_{2}, 23{ }^{\circ} \mathrm{C}, 5 \mathrm{~h}(96 \%)$. 
Table 1.

Allylation of acetate derivatives with various Lewis acids ${ }^{a}$

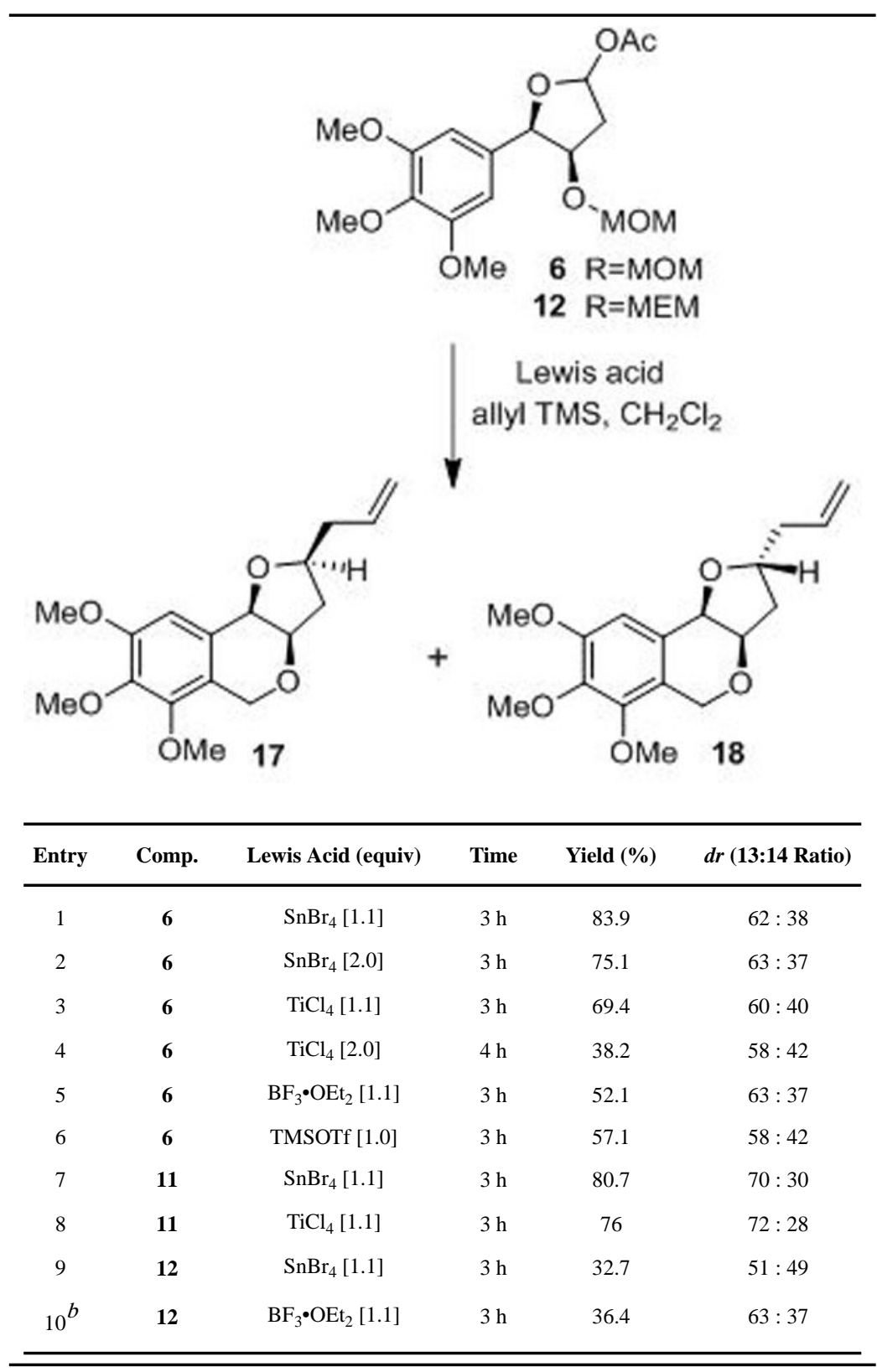

${ }^{a}$ All reactions were carried out in $\mathrm{CH}_{2} \mathrm{Cl}_{2}$ at $-78{ }^{\circ} \mathrm{C}$ and allyltrimethylsilane (4.0 equiv). Ratios were determined by ${ }^{1} \mathrm{H}-\mathrm{NMR}$ analysis;

$b_{1.5}$ equivalents of allyl trimethylsilane was added instead of 4 equivalents. 
Table 2.

Lewis acids-catalyzed tandem allylation and Oxa-Pictet-Spengler cyclization of acetate derivative $\mathbf{6}$ and $\mathbf{1 2}^{a}$<smiles>[R]O[C@H]1C[C@H](OC(C)=O)O[C@H]1c1cc(OC)c(OC)c(OC)c1</smiles><smiles>[R]OC1C[C@H](CC=C)O[C@H]1c1cc(OC)c(OC)c([C@H]2O[C@H](CC=C)C[C@H]2O[R])c1</smiles>

\begin{tabular}{lllll}
\hline Entry & Comp. & $\begin{array}{l}\text { Lewis Acid } \\
\text { (equiv, time) }\end{array}$ & Yield\% (13/14) & $\begin{array}{l}\text { Yield \% } \\
(\mathbf{1 7 / 1 8} \text { and ratio) }\end{array}$ \\
\hline 1 & $\mathbf{6}$ & $\operatorname{SnBr}_{4}(1.6,4 \mathrm{~h})$ & 54 & $22(3: 1)$ \\
$2^{b}$ & $\mathbf{6}$ & $\begin{array}{l}\mathrm{SnBr}_{4}(1.6,4 \mathrm{~h}) \\
\text { then }(2,12 \mathrm{~h})\end{array}$ & -- & $52(4: 1)$ \\
$3^{c}$ & $\mathbf{6}$ & $\mathrm{SnBr}_{4}(1.1,3 \mathrm{~h})$ then $(2,12 \mathrm{~h})$ & --- & $35(3.2: 1)$ \\
$4^{d, b}$ & $\mathbf{6}$ & $\operatorname{SnBr}_{4}(1.1,3 \mathrm{~h})$ then $(2,12 \mathrm{~h})$ & --- & $42(3: 1)$ \\
$5^{c}$ & $\mathbf{6}$ & $\begin{array}{l}\operatorname{TiCl}_{4}(1,4 \mathrm{~h}) \\
\operatorname{then}(1.5,12 \mathrm{~h})\end{array}$ & trace & trace \\
$6^{b}$ & $\mathbf{1 2}$ & $\operatorname{SnBr}_{4}(1.6,3 \mathrm{~h})$, then $(2,12 \mathrm{~h})$ & --- & $21(1.7: 1)$ \\
\hline
\end{tabular}

${ }^{a}$ All reactions were carried out in $\mathrm{CH}_{2} \mathrm{Cl}_{2}$ with 4 equiv of allyltrimethylsilane at $-78{ }^{\circ} \mathrm{C}$ to $23{ }^{\circ} \mathrm{C}$;

${ }^{b}$ Additional Lewis acid was added at $0{ }^{\circ} \mathrm{C}$ and the reaction mixture was slowly warmed to $23^{\circ} \mathrm{C}$;

${ }^{c}$ Additional Lewis acid was added at $-78^{\circ} \mathrm{C}$ and the reaction mixture was warmed to $23^{\circ} \mathrm{C}$;

${ }^{d}$ After adding 1.1 equivalents of Lewis acid, the reaction mixture was stirred at $-78^{\circ} \mathrm{C}$ for $3 \mathrm{~h}$. 